\title{
ON THE COEFFICIENT GROUPS OF EQUIVARIANT $K$-THEORY
}

\author{
YIMIN YANG
}

\begin{abstract}
We calculated the coefficient groups of equivariant $K$-theory for any cyclic group, and we proved that, for any compact Lie group, the coefficient groups can only have 2-torsion. If there is any 2-torsion, it is detected by 2primary finite subgroups of $G$. The rationalization of the coefficient groups then can be easily calculated.
\end{abstract}

\section{INTRODUCTION}

Equivariant cohomology theory plays an important role in algebraic topology. It is not a simple analogue of ordinary cohomology theory. Equivariant $\boldsymbol{K}$ theory is probably the best known example and has been studied for a number of years. One of the significant differences between an ordinary cohomology theory and an equivariant cohomology theory is that the coefficient ring of the latter is indexed by all virtual real representations $[3,4]$. Throughout this paper, $G$ will denote a finite group and $|G|$ its order. $\boldsymbol{K}_{G}$ will denote equivariant $\boldsymbol{K}$ theory defined over all $G$-CW complexes. Let $\widetilde{\boldsymbol{K}}_{G}^{*}\left(\boldsymbol{S}^{0}\right)=\widetilde{\boldsymbol{K}}_{G}\left(\boldsymbol{S}^{*}\right)$ be the set consisting of all $\widetilde{\boldsymbol{K}}_{G}\left(\boldsymbol{S}^{V}\right)$ as $\boldsymbol{V}$ runs through all the virtual real representations of $G$. Here, if $\boldsymbol{V}$ is an honest representation, $\boldsymbol{S}^{\boldsymbol{V}}$ denotes the one-point compactification of $\boldsymbol{V}$. For a virtual representation $\boldsymbol{V}-W, \boldsymbol{S}^{\boldsymbol{V}-\boldsymbol{W}}$ must be interpreted as a $G$-spectrum. However, since $S^{V-W} \wedge S^{2 W}=S^{V+W}$ and $S^{2 W}$ is complex, it will suffice to focus attention on the $\boldsymbol{S}^{V}$ for honest representations. One of the primary problems is then the determination of this coefficient ring $\widetilde{\boldsymbol{K}}_{G}^{*}\left(\boldsymbol{S}^{0}\right)$. Besides the famous Bott periodicity theorem [7] the calculations of $\boldsymbol{K}_{G}$-groups are surprisingly difficult. The Bott periodicity theorem tells us that $\widetilde{\boldsymbol{K}}_{G}\left(\boldsymbol{S}^{V}\right) \cong \boldsymbol{R}(G)$ for a complex representation $\boldsymbol{V}$ of $G$, where $\boldsymbol{R}(G)$ is the complex representation ring of $G$. This, of course, gives a partial solution to the problem. However, since the coefficient ring is $\boldsymbol{R O}(G)$-graded we have to ask what happens when $V$ is a real representation of $G$. We can actually get some help from the Bott periodicity theorem. If $\boldsymbol{V}=\boldsymbol{V}^{\prime} \oplus \boldsymbol{W}$ for some complex representation $W$ of $G$, then we have an isomorphism

$$
\widetilde{\boldsymbol{K}}_{G}\left(\boldsymbol{S}^{\boldsymbol{V}}\right)=\widetilde{\boldsymbol{K}}_{G}\left(\boldsymbol{S}^{\boldsymbol{V}^{\prime} \oplus \boldsymbol{W}}\right)=\widetilde{\boldsymbol{K}}_{G}\left(\boldsymbol{S}^{\boldsymbol{V}^{\prime}} \wedge \boldsymbol{S}^{W}\right) \cong \widetilde{\boldsymbol{K}}_{G}\left(\boldsymbol{S}^{\boldsymbol{V}^{\prime}}\right) \text {. }
$$

It is therefore sufficient to work with those real representations which contain no complex representations (I will call them pure real representations). Notice that if $|G|$ is odd, due to the lack of nontrivial pure representations, the Bott

Received by the editors May 24, 1993.

1991 Mathematics Subject Classification. Primary 19L47; Secondary 55N91, 55N25, 55N15. 
periodicity theorem does provide the answer for the problem. $\widetilde{\boldsymbol{K}}_{G}\left(\boldsymbol{S}^{\boldsymbol{V}}\right)$ is either $\boldsymbol{R}(G)$ or 0 depending on the dimension of $\boldsymbol{V}$.

In this paper we shall provide results both on the torsion elements (if any) and nontorsion elements of $\widetilde{\boldsymbol{K}}_{G}\left(\boldsymbol{S}^{\boldsymbol{V}}\right)$ where $\boldsymbol{V}$ is any real representation of $G$. We begin with a result of James McClure [5, Proposition 2.1].

Proposition 1. Suppose that $G$ is a finite group, $n=|G|$, and $X$ is a finite $G-C W$ complex. Then restrictions to cyclic subgroups induce a monomorphism:

$$
\boldsymbol{K}_{G}(X)\left[\frac{1}{n}\right] \hookrightarrow \prod_{H} K_{H}(X)\left[\frac{1}{n}\right],
$$

where $H$ runs through all cyclic subgroups of $G$.

More precisely, $\boldsymbol{K}_{G}(X)\left[\frac{1}{n}\right]$ is the inverse limit of $\boldsymbol{K}_{H}(X)\left[\frac{1}{n}\right]$.

The reason this proposition holds is that the map

$$
\sum_{H} \operatorname{tr}_{H}^{G}: \sum_{H} \boldsymbol{R}(H)\left[\frac{1}{n}\right] \rightarrow \boldsymbol{R}(G)\left[\frac{1}{n}\right]
$$

is surjective by the Brauer induction theorem. Here $H$ runs through all cyclic subgroups of $G$ and $\operatorname{tr}_{H}^{G}$ is the transfer map.

Corollary 2. Suppose that $G$ is a finite group, $n=|G|$, and $X$ is a finite $G$-CW complex. If for every cyclic subgroup $H$ of $G, K_{H}(X)$ has no p-torsion for all $p$ not dividing $n$, then $\boldsymbol{K}_{G}(X)$ has no p-torsion for all $p$ not dividing $n$.

Our study first considers the case when the group $G$ is cyclic. Because any odd-order cyclic group has no nontrivial pure real representations, we assume that our group is $\mathbb{Z}_{m}$ with $m=2 q$ an even number. It is easy to see that the group $\mathbb{Z}_{m}$ has only one nontrivial one-dimensional pure real representation $\lambda$ and one nontrivial two-dimensional pure real representation $\lambda \oplus \mathbf{1}$. Here the action of the generator of $\mathbb{Z}_{m}$ on $\lambda$ is multiplication by -1 . We shall calculate $\widetilde{\boldsymbol{K}}_{\mathbb{Z}_{m}}\left(\boldsymbol{S}^{\lambda}\right)$ and $\widetilde{\boldsymbol{K}}_{\mathbb{Z}_{m}}\left(\boldsymbol{S}^{\lambda \oplus \mathbf{1}}\right)$ in $\S \S 2$ and 3, respectively. Here are the results.

Let $\chi_{l}$ be the one-dimensional complex representation of $\mathbb{Z}_{m}$ such that the action of the generator is multiplication by $e^{2 \pi l i / m}$.

\section{MAIN RESUltS}

\section{Theorem A.}

$$
\widetilde{\boldsymbol{K}}_{\mathbb{Z}_{m}}\left(\boldsymbol{S}^{\lambda}\right) \cong \boldsymbol{R}\left(\mathbb{Z}_{m}\right)\left(\chi_{q}-1\right),
$$

where $\boldsymbol{R}\left(\mathbb{Z}_{m}\right)\left(\chi_{q}-1\right)$ denotes the ideal in $\boldsymbol{R}\left(\mathbb{Z}_{m}\right)$ generated by $\chi_{q}=1$.

Theorem B.

$$
\widetilde{\boldsymbol{K}}_{\mathbb{Z}_{m}}\left(\boldsymbol{S}^{\lambda \oplus 1}\right) \cong \boldsymbol{R}\left(\mathbb{Z}_{q}\right)\left(\chi_{2}-1\right) .
$$

In particular, the groups in $\widetilde{\boldsymbol{K}}_{\mathbb{Z}_{m}}\left(\boldsymbol{S}^{*}\right)$ are of one of the following forms:

$$
\boldsymbol{R}\left(\mathbb{Z}_{m}\right), \quad \boldsymbol{R}\left(\mathbb{Z}_{m}\right)\left(\chi_{1}-1\right), \quad \boldsymbol{R}\left(\mathbb{Z}_{q}\right)\left(\chi_{2}-1\right), \quad \text { and } 0 .
$$

Notice that $\widetilde{\boldsymbol{K}}_{\mathbb{Z}_{m}}\left(\boldsymbol{S}^{*}\right)$ is torsion free. Applying Proposition 1, we have the following 
Corollary C. The only possible torsion in $\widetilde{\boldsymbol{K}}_{G}\left(\boldsymbol{S}^{*}\right)$ is p-torsion for primes $p$ dividing $n$ and the nontorsion part of $\boldsymbol{K}_{G}\left(\boldsymbol{S}^{*}\right)$ is completely determined by Theorem $A$ and Theorem $B$ through the isomorphism

$$
\widetilde{\boldsymbol{K}}_{G}\left(\boldsymbol{S}^{*}\right)\left[\frac{1}{n}\right] \cong \underset{\mathbb{Z}_{m} \subset G}{\lim } \widetilde{\boldsymbol{K}}_{\mathbb{Z}_{m}}\left(\boldsymbol{S}^{*}\right)\left[\frac{1}{n}\right],
$$

where $H$ runs through the cyclic subgroups of $G$.

In the same paper mentioned above, James McClure also proved a beautiful theorem concerning restrictions to elementary subgroups. More precisely, we call a group an elementary group if it is a product of a $p$-group and a cyclic group having order prime to $p$.

Theorem 3 (James McClure). Suppose that $G$ is a compact Lie group and $X$ a finite $G-C W$ complex. Then restrictions to finite elementary subgroups induce a monomorphism:

$$
\boldsymbol{K}_{G}(X) \hookrightarrow \prod_{H} \boldsymbol{K}_{H}(X),
$$

where $H$ runs through all the finite elementary subgroups.

We therefore study the case where the group $G$ is an elementary group. The first thing we will prove is a lemma on the pure real representations of elementary groups.

Lemma D. Suppose $G=\mathbb{Z}_{m} \times P$, where $P$ is a p-group. Let $\mathrm{V}$ be a pure real representation of $G$.

1. If $p$ is odd, then $\mathrm{v}=\boldsymbol{\alpha} \otimes \mathbf{1}$. Here $\boldsymbol{\alpha}$ is a pure real representation of $\mathbb{Z}_{m}$.

2. If $m$ is odd, then $\mathbf{V}=\mathbf{1} \otimes \boldsymbol{\alpha}$. Here $\boldsymbol{\alpha}$ is a pure real representation of $P$.

In particular, if $G$ is an elementary group and $P$ a 2-group, then every pure real representation is of the form $1 \otimes \boldsymbol{\alpha}$.

The following theorem discusses the possible torsion elements and reduces the problem to that of computing the torsion of $\widetilde{\boldsymbol{K}}_{H}\left(\boldsymbol{S}^{*}\right)$ where $H$ is a 2-subgroup of $G$.

Theorem E. If $P$ is p-group with $p$ odd, and $Q$ is a 2-group, then

1. $\widetilde{\boldsymbol{K}}_{\mathbb{Z}_{m} \times P}\left(\boldsymbol{S}^{*}\right)$ is torsion-free.

2. $\widetilde{\boldsymbol{K}}_{\mathbb{Z}_{m} \times Q}\left(\boldsymbol{S}^{*}\right)$ can only have 2-torsion.

As an immediate corollary of Theorems $\mathrm{E}$ and Theorem 3, we have

Theorem F. $\widetilde{\boldsymbol{K}}_{G}\left(\boldsymbol{S}^{*}\right)$ can only have 2-torsion. In particular if $\widetilde{\boldsymbol{K}}_{H}\left(\boldsymbol{S}^{*}\right)$ is torsionfree for all 2-subgroups $H$, so is $\widetilde{\boldsymbol{K}}_{G}\left(\boldsymbol{S}^{*}\right)$.

For example, if $|G|=2(2 k+1)$, then $\widetilde{\boldsymbol{K}}_{G}\left(\boldsymbol{S}^{*}\right)$ is torsion-free.

We shall prove Theorem $A$ in $\S 2$ and Theorem $B$ in $\S 3$. Lemma $D$ and Theorem $E$ will be proved in $\S 4$.

I would like to thank the referee for suggesting a result of Graeme Segal which greatly reduced the size of the last section. The author also wants to thank Peter May for his invaluable advice. I am very grateful to Gustavo Comezana for the time he spent carefully reading and criticizing the drafts.

\section{THE PROOF OF THEOREM A}

Throughout this section and the next, $G=\mathbb{Z}_{m}$ will be a cyclic group of order $m=2 q$. Bundles will mean complex vector bundles. Fix a generator $g$ 
in $G$. Let $\lambda$ be the unique nontrivial one-dimensional real representation of $\mathbb{Z}_{m}$. Then the action of $\mathbb{Z}_{m}$ on $\lambda$ is given by $\mathfrak{g}^{k} t=(-1)^{k} t$ for $t \in \lambda$. If we identify $S^{\lambda}$ with $S^{1}$, the action of $\mathbb{Z}_{m}$ then is given by

$$
\mathfrak{g}^{k} z= \begin{cases}z, & k \text { even }, \\ \bar{z}, & k \text { odd },\end{cases}
$$

for $z \in S^{1}$. It is easy to see that $\lambda$ is the unique nontrivial irreducible pure real representation. To determine $\widetilde{\boldsymbol{K}}_{\mathbb{Z}_{m}}\left(\boldsymbol{S}^{*}\right)$ is then to calculate $\widetilde{\boldsymbol{K}}_{\mathbb{Z}_{m}}\left(\boldsymbol{S}^{\lambda}\right)$ and $\widetilde{\boldsymbol{K}}_{\mathbb{Z}_{m}}\left(S^{1 \oplus \lambda}\right)$. We shall prove Theorem A in this section.

Let $X$ be any $\mathbb{Z}_{m}$-space. Suppose that $\eta$ and $\zeta$ are two equivariant bundles over $X$. We shall write $\eta \doteq \zeta$ if $\eta$ and $\zeta$ are nonequivariantly isomorphic. If $\eta$ is nonequivariantly trivial, then $\eta \doteq X \times \mathbb{C}^{n}$ for some $n$. In the case where $X=S^{\lambda}$, every bundle is nonequivariantly trivial. Because our group is cyclic, the action of the group is completely determined by the action of the generator $\mathfrak{g}$. For $x \in X, \mathfrak{g}$ maps the fiber $\eta_{x}$ to the fiber $\eta_{\mathfrak{g} x}$. This action can be described by a matrix $E(x) \in U(n)$. That is,

$$
\mathfrak{g}(x, u)=(\mathfrak{g} x, E(x) u)
$$

for $(x, u) \in \eta \doteq X \times \mathbb{C}^{n}$. The action of $\mathfrak{g}^{k}$ is given by the matrix $\prod_{i=0}^{k-1} E\left(\mathfrak{g}^{i} x\right)$. Since the order of $\mathfrak{g}$ is $m$, we have $\prod_{i=0}^{m-1} E\left(\mathfrak{g}^{i} x\right)=I$ with $I$ understood to be the identity matrix. The main object of our attention in this section is called a bracket. It should be viewed as another way of describing nonequivariantly trivial $\mathbb{Z}_{m}$-bundles. A bracket, denoted $[n \mid E]$, consists of the dimension $n$ and the action $E$ of $\mathfrak{g}$.

\section{Definition 2.1.}

1. Bracket: $[n \mid E]$ is a bracket over $X$ if $E: X \rightarrow U(n)$ is a continuous function satisfying

$$
\prod_{i=0}^{m-1} E\left(\mathfrak{g}^{i} x\right)=I
$$

for all $x \in X$, where $I$ is the identity matrix in $U(n)$.

2. The associated bundle $\Theta[n \mid E]$ of a bracket: We may recover $\mathbb{Z}_{m}$-bundles from brackets. Namely, define its associated $\mathbb{Z}_{m}$-bundle $\Theta[n \mid E]$ over $X$ by setting

$$
\Phi[n \mid E]=X \times \mathbb{C}^{n} \stackrel{\text { Proj. }}{\rightarrow} X
$$

where the action of $G$ is given by

$$
\mathfrak{g}(x, u)=(\mathfrak{g} x, E(x) u)
$$

for all $x \in X$ and $u \in \mathbb{C}^{n}$.

3. Isomorphism of brackets: Two brackets $[n \mid E]$ and $[r \mid K]$ are said to be isomorphic by $\Phi$ (denoted $[n \mid E] \cong[r \mid K])$ if $n=r$ and $\Phi: X \rightarrow U(n)$ is a continuous function satisfying $K(x) \Phi(x)=\Phi(\mathfrak{g} x) E(x)$ for all $x \in$ $X$.

4. We may also define homotopic brackets; direct sums of brackets; constant (trivial) brackets; stable homotopic and stable isomorphic brackets; etc. in obvious ways. We notice that homotopic brackets give isomorphic bundles. 
Definition 2.2. From now on, let $X=S^{\lambda}$ with $\mathbb{Z}_{m}$ acting on $S^{\lambda}$ by

$$
\mathfrak{g}^{k} z= \begin{cases}z, & k \text { even }, \\ \bar{z}, & k \text { odd. }\end{cases}
$$

1. $\boldsymbol{D}(z)$ function: If $[n \mid E]$ is a bracket over $\boldsymbol{S}^{\lambda}$, define an auxiliary function $D(z)$ as follows:

$$
D(z)=E(\bar{z}) E(z) \text { for } z \in S^{\lambda} .
$$

The function $D(z)$ represents the action of $\mathfrak{g}^{2}$. Notice that $D^{q}(z)=I$ by the definition of a bracket.

2. Special brackets: A bracket $[n \mid E]$ is said to be special if $D(z)$ is constant over $\boldsymbol{S}^{\lambda}$.

3. Characteristic numbers: Let $S^{0}=\{ \pm 1\} \subset S^{\lambda}$ be the fixed point set of $S^{\lambda} \cdot \mathfrak{g}( \pm 1)= \pm 1$. For any bracket $[n \mid E]$, define its characteristic number $\Omega[n \mid E]$ as

$$
\Omega[n \mid E]=\frac{\operatorname{det}(t I-E(+1))}{\operatorname{det}(t I-E(-1))},
$$

where $t$ is an indeterminate.

It is easy to see that the range of this characteristic number is in the field of rational functions $\mathbb{C}(t)$.

Proposition 2.3. 1. $\Omega([n \mid E] \oplus[r \mid K])=\Omega[n \mid E] \cdot \Omega[r \mid K]$.

2. $\Omega[n \mid E]=1 \Leftrightarrow E(+1)$ is similar to $E(-1)$.

In particular, if $[n \mid E]$ is a constant bracket, then $\Omega[n \mid E]=1$.

Lemma 2.4. 1. Let $A$ be a matrix in $U(n)$. Then the centralizer of $A$ in $U(n)$ is connected.

2. The map $\boldsymbol{S}^{1} \rightarrow \boldsymbol{U}(2)$ defined by $z \rightarrow\left(\begin{array}{ll}\bar{z} & 0 \\ 0 & z\end{array}\right)$ is nullhomotopic.

3. Any continuous map $f: S^{1} \rightarrow U(n)$ is homotopic to a map $z \rightarrow\left(\begin{array}{cc}I & 0 \\ 0 & z^{k}\end{array}\right)$ for some $k \in \mathbb{Z}$.

4. The map $\boldsymbol{S}^{1} \rightarrow \boldsymbol{U}(n)$ defined by

$$
z \rightarrow\left(\begin{array}{ccc}
z^{r_{1}} & \cdots & 0 \\
\vdots & \ddots & \vdots \\
0 & \cdots & z^{r_{n}}
\end{array}\right) \quad\left(r_{1}, \ldots, r_{n} \in \mathbb{Z}\right)
$$

is homotopic to the maps

$$
z \rightarrow\left(\begin{array}{cc}
z^{r} & 0 \\
0 & I
\end{array}\right) \text { and } z \rightarrow\left(\begin{array}{cc}
I & 0 \\
0 & z^{r}
\end{array}\right)
$$

where $r=\sum r_{i}$.

Proof. 1. As a unitary matrix, $A=S D \bar{S}^{-t}$, where $S$ is unitary and

$$
D=\left(\begin{array}{ccc}
e^{i t_{1}} & \cdots & 0 \\
\vdots & \ddots & \vdots \\
0 & \cdots & e^{i t_{n}}
\end{array}\right)
$$

is diagonal. The centralizer of $A$ is conjugate to the centralizer of $D$ by $\bar{S}^{t}$ and the latter is obviously connected. 
2. It suffices to prove that

$$
\left(\begin{array}{cc}
\bar{z}^{2} & 0 \\
0 & z^{2}
\end{array}\right)
$$

is nullhomotopic. The following is such a homotopy:

$$
\left(\begin{array}{cc}
\cos \frac{\pi}{2} t & \sin \frac{\pi}{2} t \\
-\sin \frac{\pi}{2} t & \cos \frac{\pi}{2} t
\end{array}\right) \cdot\left(\begin{array}{ll}
\bar{z} & 0 \\
0 & z
\end{array}\right) \cdot\left(\begin{array}{cc}
\cos \frac{\pi}{2} t & -\sin \frac{\pi}{2} t \\
\sin \frac{\pi}{2} t & \cos \frac{\pi}{2} t
\end{array}\right) \cdot\left(\begin{array}{cc}
\bar{z} & 0 \\
0 & z
\end{array}\right) .
$$

3. This is because $\pi_{1}(U(n))=\mathbb{Z}$.

4. Both maps have the same degree.

Lemma 2.5. If $[n \mid E]$ is a bracket over $\boldsymbol{S}^{1}$, then there is a diagonal matrix $\Lambda_{0}$ in $\boldsymbol{U}(r)$ satisfying $\Lambda_{0}^{2 q}=I$ for some $r \in \mathbb{Z}$ and a special bracket $\left[(n+r+1) \mid H_{0}\right]$ such that $[n \mid E] \oplus\left[r \mid \Lambda_{0}\right] \oplus\left[1 \mid z^{2 k}\right]$ is homotopic to $\left[(n+r+1) \mid H_{0}\right]$, where $\left[r \mid \Lambda_{0}\right]$ is constant. In particular,

$$
\Theta[n \mid E] \oplus \Theta\left[r \mid \Lambda_{0}\right] \oplus \Theta\left[1 \mid z^{2 k}\right] \cong \Theta\left[(n+r+1) \mid H_{0}\right]
$$

as $\mathbb{Z}_{m}$-bundles over $\boldsymbol{S}^{\lambda}$.

Proof. Let $D(z)=E(\bar{z}) E(z)$ be the auxiliary function. We have already observed that $D^{q}(z)=I$.

Claim: There is an isomorphism $\Delta: S^{1} \rightarrow U(n+r)$ such that

$$
\left(\begin{array}{cc}
D(z) & 0 \\
0 & D_{2}
\end{array}\right)=\Delta^{-1}(z)\left(\begin{array}{cc}
D_{1} & 0 \\
0 & D_{2}
\end{array}\right) \Delta(z)
$$

with $D_{1}, D_{2}$ diagonal, $D_{1}^{q}=I$, and $D_{2}^{q}=I$.

Let $\eta=S^{\lambda} \times \mathbb{C}^{n} \rightarrow S^{\lambda}$ be a $\mathbb{Z}_{m}$-bundle over $S^{1}$. The action of the generator $\mathfrak{g}$ is given by $\mathfrak{g}(z, u)=(z, E(z) u)$. Let $\mathbb{Z}_{q}$ be the cyclic group of order $q$ generated by $\mathfrak{b}=\mathfrak{g}^{2}$. Considered as a subgroup of $\mathbb{Z}_{m}$ by mapping $\mathfrak{b}$ to $\mathfrak{g}^{2}, \mathbb{Z}_{q}$ acts trivially on $\boldsymbol{S}^{\lambda}$. $\eta$ restricts to a $\mathbb{Z}_{q}$-bundle over $\boldsymbol{S}^{1}$ with action $\mathfrak{b}(z, u)=(z, D(z) u)$.

Since $\widetilde{\boldsymbol{K}}_{\mathbb{Z}_{q}}\left(\boldsymbol{S}^{1}\right)=0$, there are representations $V$ and $\boldsymbol{W}$ of $\mathbb{Z}_{q}$ such that $\eta \oplus \boldsymbol{V} \simeq \boldsymbol{W} \oplus \boldsymbol{V}$ as $\mathbb{Z}_{q}$-bundles over $\boldsymbol{S}^{1}$. Here $\simeq$ means $\mathbb{Z}_{q}$-bundle isomorphism.

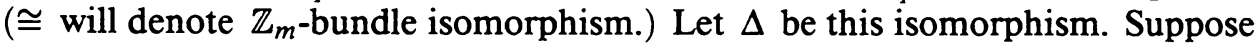
$\mathfrak{b} \in \mathbb{Z}_{q}$ acts on $\boldsymbol{W}$ and $\boldsymbol{V}$ through left multiplication by $D_{1} \in \boldsymbol{U}(n)$ and $D_{2} \in \boldsymbol{U}(r)$, respectively. We may assume that $D_{1}$ and $D_{2}$ are diagonal. Then there is a map $\Delta: S^{1} \rightarrow U(n+r)$ such that

$$
\begin{array}{ccc}
\eta \oplus \boldsymbol{V} & \cong & \boldsymbol{W} \oplus \boldsymbol{V} \\
\| \cdot & \| \cdot \\
\boldsymbol{S}^{1} \times \mathbb{C}^{n} \longrightarrow \boldsymbol{S}^{1} \times(\boldsymbol{W} \oplus \boldsymbol{V}) \\
(z,(u, v)) \longrightarrow\left(z, \Delta(z)\left(\begin{array}{c}
u \\
v
\end{array}\right)\right)
\end{array}
$$

It follows from the fact that $\Delta$ is $\mathbb{Z}_{q}$-equivariant that

$$
\Delta(z)\left(\begin{array}{cc}
D(z) & 0 \\
0 & D_{2}
\end{array}\right)=\left(\begin{array}{cc}
D_{1} & 0 \\
0 & D_{2}
\end{array}\right) \Delta(z) .
$$

This proves our claim. 
Suppose that $\Delta: S^{1} \rightarrow U(n+r)$ is homotopic to

$$
z \rightarrow\left(\begin{array}{cc}
I & 0 \\
0 & z^{k}
\end{array}\right)
$$

for some $k \in \mathbb{Z}$ through some homotopy $\Delta_{t}$. Set

$$
\Psi(z)=\left(\begin{array}{cc}
\Delta(z) & 0 \\
0 & 1
\end{array}\right)
$$

Then $\Psi(z)$ is homotopic to $\left(\begin{array}{cc}I & 0 \\ 0 & z^{k}\end{array}\right)$ in $U(n+r+1)$ for some $k$ through $\Psi_{t}(z)$. Pick a diagonal matrix $\Lambda_{0}$ with $\Lambda_{0}^{2}=D_{2}$ and define a homotopy $H$ by setting

$$
H(t, z)=\Psi_{t}(\bar{z})\left(\begin{array}{ccc}
E(z) & 0 & 0 \\
0 & \Lambda_{0} & 0 \\
0 & 0 & 1
\end{array}\right) \Psi_{t}^{-1}(z),
$$

where $z \in \boldsymbol{S}^{1}$. Then direct calculation shows that

$$
(H(t, \bar{z}) H(t, z))^{q}=I .
$$

$H$ gives a homotopy between $\Theta\left[(n+r+1) \mid H_{0}\right]$ and $\left[(n+r+1) \mid H_{1}\right]$. We want to find out what kind of brackets $\left[n+r+1 \mid H_{0}\right]$ and $\left[n+r+1 \mid H_{1}\right]$ are. By definition of $H$ we find

$$
H_{0}(z)=H(0, z)=\left(\begin{array}{cc}
\Delta(\bar{z})\left(\begin{array}{cc}
E(z) & 0 \\
0 & \Lambda_{0}
\end{array}\right) \Delta^{-1}(z) & 0 \\
& 0
\end{array}\right),
$$

so that

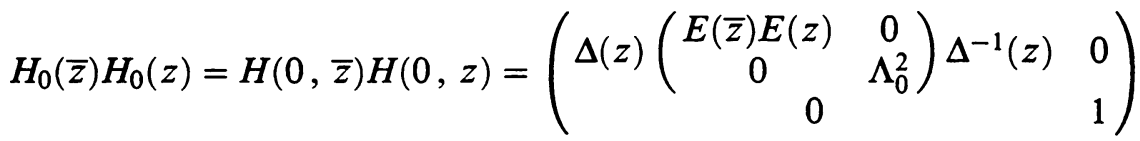

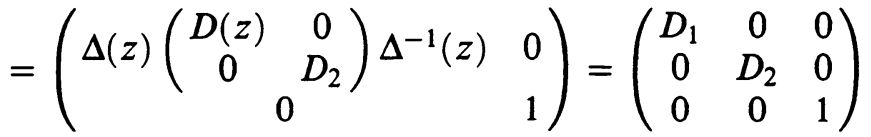

which is of order $q$.

This proves that $\left[(n+r+1) \mid H_{0}\right]$ is a special bracket. On the other hand,

$$
H_{1}(z)=\left(\begin{array}{ccc}
E(z) & 0 & 0 \\
0 & \Lambda_{0} & 0 \\
0 & 0 & z^{2 k}
\end{array}\right)
$$

Therefore

$$
\left[(n+r+1) \mid H_{1}\right]=[n \mid E] \oplus\left[r \mid \Lambda_{0}\right] \oplus\left[1 \mid z^{2 k}\right]
$$

is homotopic to $\left[(n+r+1) \mid H_{0}\right]$. Q.E.D.

Lemma 2.6. 1. Let $[n \mid E]$ be a special bracket over $\boldsymbol{S}^{\lambda}$. Then $[n \mid E]$ is homotopic to some special bracket $\left[n \mid K_{1}\right]$ such that $K_{1}(+1)$ is diagonal.

2. If $[n \mid E]$ is special with $E(+1)$ diagonal, then $[n \mid E] \cong[n \mid K]$, where

$$
K(z)=\left(\begin{array}{cc}
I_{r} & 0 \\
0 & z I_{s}
\end{array}\right) E(+1),
$$

and $[n \mid K]$ is a special bracket. 
In particular, $\Theta[n \mid E] \cong \Theta[n \mid K]$ and

$$
[n \mid E] \cong\left[1 \mid \lambda_{1}\right] \oplus \cdots \oplus\left[1 \mid \lambda_{r}\right] \oplus\left[1 \mid z \lambda_{r+1}\right] \oplus \cdots \oplus\left[1 \mid z \lambda_{n}\right] .
$$

Here

$$
E(+1)=\left(\begin{array}{ccc}
\lambda_{1} & \cdots & 0 \\
\vdots & \ddots & \vdots \\
0 & \cdots & \lambda_{n}
\end{array}\right)
$$

Each $\left[1 \mid \lambda_{i}\right]$ is a constant bracket.

3. Let $[n \mid E]$ be any bracket. Then $[n \mid E] \oplus\left[1 \mid z^{2 k}\right]$ is stably homotopic to a sum of $[1 \mid z \lambda]$ 's, where each $\lambda$ is an mth-root of unity. In particular, $\Theta[n \mid E] \oplus \Theta\left[1 \mid z^{2 k}\right]$ is stably homotopic to a sum of $\Theta[1 \mid z \lambda]$ 's.

Proof. 1. Since $D=D(z)=E(\bar{z}) E(z)$ is a constant, $E( \pm 1)^{m}=E( \pm 1)^{2 q}=$ $D^{q}=I$, where $m=2 q$. Suppose $E(+1)=T \Lambda T^{-1}$, with $\Lambda$ diagonal and $\Lambda^{m}=I$ for some $T \in U(n)$. Let $r:[0,1] \rightarrow U(n)$ be a path in $\boldsymbol{U}(n)$ such that $r(0)=I$ and $r(1)=T$.

Define $K(t, z)=r^{-1}(t) E(z) r(t)$. Then

$$
K(t, \bar{z}) K(t, z)=r^{-1}(t) E(\bar{z}) E(z) r(t)=r^{-1}(t) \operatorname{Dr}(t) .
$$

Thus we have

$$
(F(t, \bar{z}) F(t, z))^{q}=r^{-1}(t) D^{q} r(t)=I .
$$

This proves that $[n \mid K]$ is a bracket that defines a homotopy. Moreover, $K(0, z)$ $=E(z)$, while $K(1, z)=T^{-1} E(z) T$. Hence,

$$
K(1, \bar{z}) K(1, z)=r^{-1}(t) E(\bar{z}) E(z) r(t)=T^{-1} D T=\text { constant },
$$

with

$$
K(1,+1)=r^{-1}(1) E(+1) r(1)=T^{-1} E(+1) T=\lambda \text { diagonal. }
$$

This proves that $[n \mid E]=\left[n \mid K_{0}\right]$ is homotopic to $\left[n \mid K_{1}\right]$; the latter $\left[n \mid K_{1}\right]$ is special with $K_{1}(+1)=\Lambda$ diagonal.

2. Since $D=E(\bar{z}) E(z)=$ constant, $E^{2}( \pm 1)=D$. If $E(-1)=S \Lambda S^{-1}$ with $\Lambda$ a diagonal matrix, then $E^{2}(+1)=E^{2}(-1)=S \Lambda^{2} S^{-1}$. Because $E^{2}(+1)$ is diagonal we may assume that $E^{2}(+1)=\Lambda^{2}$. Furthermore, we may assume

$$
\Lambda=\left(\begin{array}{cc}
I_{r} & 0 \\
0 & -I_{s}
\end{array}\right) E(+1)
$$

Then $D=E^{2}(-1)=S \Lambda^{2} S^{-1}=S D S^{-1}$. Accordingly, $D S=S D$. Using Lemma 2.4, we get a path $r(t)$ in $U(n)$ such that $r(0)=I, r(1)=S$, and $r(t) D=D r(t)$ for $t \in[0,1]$.

Define, for $z=e^{i \theta}$ :

$$
\begin{aligned}
\Phi\left(e^{i \theta}\right) & = \begin{cases}\left(r\left(\frac{\theta}{\pi}\right)\right)^{-1}, & 0 \leq \theta \leq \pi, \\
K(\bar{z})\left(r\left(-\frac{\theta}{\pi}\right)\right)^{-1}\left(E\left(e^{-i \theta}\right)\right)^{-1}, & -\pi \leq \theta \leq 0,\end{cases} \\
& =\left\{\begin{array}{cc}
\left(r\left(\frac{\theta}{\pi}\right)\right)^{-1}, & 0 \leq \theta \leq \pi, \\
\left(\begin{array}{cc}
I_{r} & 0 \\
0 & \bar{z} I_{s}
\end{array}\right) E(+1)\left(r\left(-\frac{\theta}{\pi}\right)\right)^{-1}\left(E\left(e^{-i \theta}\right)\right)^{-1}, & -\pi \leq \theta \leq 0 .
\end{array}\right.
\end{aligned}
$$

Here $K(z)=\left(\begin{array}{cc}I_{r} & 0 \\ 0 & \bar{z} I_{s}\end{array}\right) E(+1)$ is diagonal and special . 
It is easy to verify that $\Phi$ is well defined, $K(z) \Phi(z)=\Phi(\bar{z}) E(z)$, and $\Phi$ gives an isomorphism between $[n \mid E]$ and $[n \mid K]$.

3. This is an immediate consequence of Lemma 2.5 and the previous two statements.

Proposition 2.7. 1. For any $\mathbb{Z}_{m}$-bundle $\eta$ over $\boldsymbol{S}^{\lambda}$ there is a bracket $[n \mid E]$ over $S^{\lambda}$ such that $\eta \cong \Theta[n \mid E]$.

2. $\left[1 \mid z^{k}\right] \cong\left[1 \mid \bar{z}^{k}\right]$.

3. $\left[1 \mid z^{k}\right] \cong\left[1 \mid z^{k-2}\right]$.

4.

$$
\left[1 \mid z^{k}\right] \cong \begin{cases}{[1 \mid z],} & k \text { odd } \\ {[1 \mid 1],} & k \text { even }\end{cases}
$$

5. The bundle $\Theta[1 \mid z \lambda] \cong \lambda \cdot \Theta[1 \mid z]$, where $\lambda$ is the one-dimensional representation of $\mathbb{Z}_{m}$.

6. Any $\mathbb{Z}_{m}$-bundle $\eta$ over $S^{\lambda}$ is stably isomorphic to a sum of bundles $\lambda \cdot \Theta[1 \mid z]$ with $\lambda^{m}=1$.

Proof. 1. Every bundle over $\boldsymbol{S}^{1}$ is nonequivariantly trivial. So any $\mathbb{Z}_{m}$-bundle over $S^{1}$ can be recovered from a bracket.

2. Let $K(z)=\bar{z}^{k}, E(z)=z^{k}$, and $\Phi(z)=z^{k}$. Then

$$
K(z) \Phi(z)=\Phi(\bar{z}) E(z)=I .
$$

This implies that $\Phi$ gives an isomorphism $[1 \mid E] \cong[1 \mid K]$.

3. Let $K(z)=z^{k}, E(z)=z^{k-2}$, and $\Phi(z)=\bar{z}$. Then $K(z) \Phi(z)=$ $\Phi(\bar{z}) E(z)=z^{k-1}$, and thus $[1 \mid K] \cong[1 \mid E]$ by $\Phi$.

Corollary 2.8. Let $\delta=\Theta[1 \mid z]$ be the $\mathbb{Z}_{m}$-bundle associated to the bracket $[1 \mid z]$, so that $\delta \doteq \boldsymbol{S}^{\lambda} \times \mathbb{C}$ is nonequivariantly trivial. Then $\boldsymbol{K}_{\mathbb{Z}_{m}}\left(\boldsymbol{S}^{\lambda}\right)$ is generated as an $\boldsymbol{R}\left(\mathbb{Z}_{m}\right)$-module by $\delta$ together with trivial $\mathbb{Z}_{m}$-bundles. $\widetilde{\boldsymbol{K}}_{\mathbb{Z}_{m}}\left(\boldsymbol{S}^{\lambda}\right)$ is generated as an $\boldsymbol{R}\left(\mathbb{Z}_{m}\right)$-module by $\omega=1-\delta$.

We have defined the characteristic number for any bracket. We now are able to define its analogue for any $\mathbb{Z}_{m}$-bundle over $S^{\lambda}$ :

Definition 2.9. Let $\eta$ be any $\mathbb{Z}_{m}$-bundle over $S^{\lambda}$. According to Proposition 2.7, $\eta \cong \Theta[n \mid E]$ for some bracket $[n \mid E]$.

Define

$$
\Omega[\eta]=\Omega[n \mid E]=\frac{\operatorname{det}(t I-E(+1))}{\operatorname{det}(t I-E(-1))}
$$

and call $\Omega[\eta]$ the characteristic number of $\eta$.

Proposition 2.10. 1. $\Omega[\eta]$ is well defined.

2. $\Omega: K_{\mathbb{Z}_{m}}\left(\boldsymbol{S}^{\lambda}\right) \rightarrow \mathbb{C}(t)$ is "exponential"; that is,

$$
\Omega[\eta \oplus \xi]=\Omega[\eta] \cdot \Omega[\xi] .
$$

3. If $\eta$ is trivial, then $\Omega[\eta]=1$.

Lemma 2.11.

$$
\begin{aligned}
& {[1 \mid z] \oplus[1 \mid-z] \oplus[1 \mid 1] \oplus[1 \mid-1]} \\
& \quad \cong[1 \mid 1] \oplus[1 \mid-1] \oplus[1 \mid 1] \oplus[1 \mid-1] .
\end{aligned}
$$


Proof. Let $\alpha:[0,2 \pi] \rightarrow[0,2 \pi]$ be a continuous map such that $\alpha(0)=0, \alpha(\pi)$ $=\frac{\pi}{2}$, and $\alpha(2 \pi)=2 \pi$. Define

$$
\begin{gathered}
\Phi_{1}\left(e^{i \theta}\right)=\left(\begin{array}{cc}
\cos \alpha(\theta) & \sin \alpha(\theta) \\
-\sin \alpha(\theta) & \cos \alpha(\theta)
\end{array}\right), \quad 0 \leq \theta \leq 2 \pi, \\
\Phi_{1}\left(e^{i \theta}\right)=\left(\begin{array}{cc}
\cos \alpha(\theta) & \sin \alpha(\theta) \\
-\sin \alpha(\theta) & \cos \alpha(\theta)
\end{array}\right), \quad 0 \leq \theta \leq \pi, \\
e^{-i \theta}\left(\begin{array}{cc}
1 & 0 \\
0 & -1
\end{array}\right) \Phi_{1}\left(e^{-i \theta}\right)\left(\begin{array}{cc}
1 & 0 \\
0 & -1
\end{array}\right) \\
=e^{-i \theta}\left(\begin{array}{cc}
\cos \alpha(-\theta) & -\sin \alpha(-\theta) \\
\sin \alpha(-\theta) & \cos \alpha(-\theta)
\end{array}\right), \quad-\pi \leq \theta \leq 0 . \\
\Phi(z)=\left(\begin{array}{cc}
0 & A(z) \\
\left(\begin{array}{ll}
1 & 0 \\
0 & 1
\end{array}\right) & 0
\end{array}\right) .
\end{gathered}
$$

It is not difficult to see that $A$ and $\Phi_{1}$ are well defined. Furthermore, $A(z)=$ $\bar{z} J A(\bar{z}) J$, where $J=\left(\begin{array}{cc}1 & 0 \\ 0 & -1\end{array}\right)$ and $z \in \boldsymbol{S}^{1}$.

$$
\left(\begin{array}{cc}
z \cdot J & 0 \\
0 & J
\end{array}\right) \Phi(z)=\Phi(\bar{z})\left(\begin{array}{ll}
J & 0 \\
0 & J
\end{array}\right) \text {. }
$$

This implies that $\Phi$ is an isomorphism between

$$
[1 \mid 1] \oplus[1 \mid-1] \oplus[1 \mid 1] \oplus[1 \mid-1]
$$

and

$$
[1 \mid z] \oplus[1 \mid-z] \oplus[1 \mid 1] \oplus[1 \mid-1] .
$$

In particular, $\Theta[1 \mid z] \oplus \Theta[1 \mid-z]$ is stably trivial. Q.E.D.

Corollary 2.12. 1. $\quad \boldsymbol{K}_{\mathbb{Z}_{m}}\left(\boldsymbol{S}^{\lambda}\right)$ is generated by trivial bundles and $\Theta\left[1 \mid \xi^{k} z\right]=$ $\xi^{k} \delta, 1 \leq k \leq q$, where $\delta=\Theta[1 \mid z]$ and $\xi=e^{2 i \pi / m}$.

2. $\widetilde{\widetilde{\boldsymbol{K}}}_{\mathbb{Z}_{m}}\left(\boldsymbol{S}^{\lambda}\right)$ is generated by $\left\{\xi^{k} \omega\right\}_{1 \leq k \leq q}$ with $\xi^{q+k} \omega=-\xi^{k} \omega$, where $\omega=$ $1-\delta$.

Theorem 2.13.

$$
\widetilde{\boldsymbol{K}}_{\mathbb{Z}_{m}}\left(\boldsymbol{S}^{\lambda}\right) \cong \mathbb{Z} \omega \oplus \mathbb{Z} \xi \omega \oplus \cdots \oplus \mathbb{Z} \xi^{q-1} \omega
$$

with $\xi^{q+k} \omega=-\xi^{k} \omega, \omega^{2}=2 \omega$.

In particular, $\widetilde{\boldsymbol{K}}_{\mathbb{Z}_{m}}\left(\boldsymbol{S}^{\lambda}\right)$ is torsion-free.

Proof. We begin by showing that $\omega^{2}=2 \omega$.

It is not difficult to see that

$$
\Theta[1 \mid z] \otimes \Theta[1 \mid z]=\Theta\left[1 \mid z^{2}\right]
$$

By applying Proposition 2.7 we get

$$
\Theta[1 \mid z] \otimes \Theta[1 \mid z] \cong \Theta[1 \mid 1]=1 .
$$

It follows then that

$$
\omega^{2}=(1-\Theta[1 \mid z]) \otimes(1-\Theta[1 \mid z])=2-2 \Theta[1 \mid z]=2 \omega .
$$

In order to prove the theorem we have to prove the following statement: If $\boldsymbol{W} \oplus n_{0} \omega \oplus n_{1} \xi \omega \oplus \cdots \oplus n_{q-1} \xi^{q-1} \omega \cong \boldsymbol{V}$, where $\boldsymbol{W}$ and $\boldsymbol{V}$ are representations of $\mathbb{Z}_{m}$ and $n_{0}, \ldots, n_{q-1} \in \mathbb{Z}$, then $n_{0}=n_{1}=\cdots=n_{q-1}=0$. 
Using the characteristic numbers above we find that

$$
\begin{aligned}
1 & =\Omega(\boldsymbol{V}) \\
& =\Omega\left(\boldsymbol{W} \oplus n_{0} \omega \oplus n_{1} \xi \omega \oplus \cdots \oplus n_{q-1} \xi^{q-1} \omega\right) \\
& =\Omega\left(n_{0} \omega \oplus n_{1} \xi \omega \oplus \cdots \oplus n_{q-1} \xi^{q-1} \omega\right) \\
& =\Omega(\omega)^{n_{0}} \cdot \Omega(\xi \omega)^{n_{1}} \cdots \Omega\left(\xi^{q-1} \omega\right)^{n_{q-1}} \\
& =\left(\frac{t-1}{t+1}\right)^{n_{0}}\left(\frac{t-\xi}{t+\xi}\right)^{n_{1}} \cdots\left(\frac{t-\xi^{q-1}}{t+\xi^{q-1}}\right)^{n_{q-1}}
\end{aligned}
$$

But this can happen only if $n_{0}=n_{1}=\cdots=n_{q-1}=0$. Q.E.D.

Remark 2.14. Let $\chi_{k} \in \boldsymbol{R}\left(\mathbb{Z}_{m}\right)$ be the one-dimensional complex representation of $\mathbb{Z}_{m}$ defined by $\mathfrak{g} z=e^{2 k \pi i / m} z$. (Here $\mathfrak{g} \in \mathbb{Z}_{m}$ is the generator.) Let $\boldsymbol{S}^{0}=$ $\{ \pm 1\} \subset S^{\lambda}$ be the fixed point set and $\omega=\Theta[1 \mid z]$. Then the Euler map

$$
\widetilde{\boldsymbol{K}}_{\mathbb{Z}_{m}}\left(\boldsymbol{S}^{\lambda}\right) \rightarrow \widetilde{\boldsymbol{K}}_{\mathbb{Z}_{m}}\left(\boldsymbol{S}^{0}\right) \cong \boldsymbol{R}\left(\mathbb{Z}_{m}\right)
$$

maps $\omega$ to $1-\chi_{q}$.

It follows that the image of $\widetilde{\boldsymbol{K}}_{\mathbb{Z}_{m}}\left(\boldsymbol{S}^{\lambda}\right)$ in $\widetilde{\boldsymbol{K}}_{\mathbb{Z}_{m}}\left(\boldsymbol{S}^{0}\right)$ is $\boldsymbol{R}\left(\mathbb{Z}_{m}\right)\left(\chi_{q}-\chi_{0}\right)$.

Finally, let us prove

Theorem A.

$$
\widetilde{\boldsymbol{K}}_{\mathbb{Z}_{m}}\left(\boldsymbol{S}^{\lambda}\right) \cong \boldsymbol{R}\left(\mathbb{Z}_{m}\right)\left(\chi_{q}-1\right)
$$

as an ideal. Moreover the Euler map

$$
\widetilde{\boldsymbol{K}}_{\mathbb{Z}_{m}}\left(\boldsymbol{S}^{\lambda}\right) \hookrightarrow \widetilde{\boldsymbol{K}}_{\mathbb{Z}_{m}}\left(\boldsymbol{S}^{0}\right)
$$

is an injection with image $\boldsymbol{R}\left(\mathbb{Z}_{m}\right)\left(\chi_{q}-\chi_{0}\right)$.

Proof. We know that $\omega=1-\Theta[1 \mid z]$ is a generator of $\widetilde{\boldsymbol{K}}_{\mathbb{Z}_{m}}\left(\boldsymbol{S}^{\lambda}\right)$ and that the Euler map sends $\omega$ to $1-\chi_{q}$. Suppose $\mu \in \boldsymbol{R}\left(\mathbb{Z}_{m}\right)$ is an element such that the Euler map maps $\mu \cdot \omega$ to 0 . Then $\mu \cdot\left(\chi_{q}-1\right)=0$ in $\boldsymbol{R}\left(\mathbb{Z}_{m}\right)$ and therefore $\mu=\nu \cdot\left(\chi_{q}+1\right)$ for some $\nu \in \boldsymbol{R}\left(\mathbb{Z}_{m}\right)$. But then

$$
\begin{aligned}
\mu \cdot \omega & =\nu \cdot\left(\chi_{q}+1\right) \cdot \omega=\nu \cdot\left(\chi_{q} \omega+\omega\right) \\
& =\nu \cdot(-\omega+\omega)=0 .
\end{aligned}
$$

\section{THE PROOF OF THEOREM B}

Previous conventions remain in force. $G=\mathbb{Z}_{m}$ is a cyclic group of even order $m=2 q$ and $\mathfrak{g} \in \mathbb{Z}_{m}$ is a generator. $\lambda$ will be the one-dimensional nontrivial real representation of $\mathbb{Z}_{m}$ with action defined by $\mathfrak{g}^{k} t=(-1)^{k} t$. Let $\mathfrak{b} \in \mathbb{Z}_{q}$ be a generator; $\mathbb{Z}_{q} \hookrightarrow \mathbb{Z}_{m}$ is embedded by mapping $\mathfrak{b}$ to $\mathfrak{g}^{2}$.

Let $S^{1}, S^{\lambda}$, and $S^{1 \oplus \lambda}$ be the one-point compactifications of $1=\mathbb{R}^{1}, \lambda$, and $1 \oplus \lambda$ respectively. The action of $\mathbb{Z}_{m}$ on $S^{\lambda}$ is $\mathfrak{g} z=\bar{z}$ for all $z \in S^{\lambda}$, while the action of $\mathbb{Z}_{q}$ is trivial. Let $D^{2}$ be the unit disc in $\mathbb{R}^{2}$ and $\partial D^{2}=S^{1}$ its boundary. Suppose that $S_{+}^{1 \oplus \lambda}$ is the right hemisphere of $S^{1 \oplus \lambda}, S_{-}^{1 \oplus \lambda}$ the left hemisphere, $\Delta_{+}$the upper hemisphere of $S^{1 \oplus \lambda}$, and $\Delta_{-}$the lower hemisphere. If $\tau: S^{1 \oplus \lambda} \rightarrow S^{1 \oplus \lambda}$ represents the action of $\mathfrak{g}$ over $S^{1 \oplus \lambda}$, then obviously $\tau$ maps $S_{ \pm}^{1 \oplus \lambda}$ to $S_{ \pm}^{1 \oplus \lambda}$ and $\Delta_{ \pm}$to $\Delta_{\mp} . S_{ \pm}^{1 \oplus \lambda}$ is $\mathbb{Z}_{m}$-invariant and contractible. If we identify $S_{ \pm}^{1 \oplus \lambda}$ with $D^{2}$, then $\tau$ is just complex conjugation. 
Since $S^{1 \oplus \lambda}$ is a 2 -sphere, not every vector bundle over $S^{1 \oplus \lambda}$ is trivial (however, we shall see that every $\mathbb{Z}_{m}$-bundle over it is nonequivariantly trivial). Our previous definition of bracket is inadequate here. We have to consider clutching functions for nontrivial bundles. Our modification of the notation of bracket will be called a clutching bracket. The point of introducing such an object is this: a $\mathbb{Z}_{m}$-bundle will be trivial when restricted to $S_{+}^{1 \oplus \lambda}$ and $S_{-}^{1 \oplus \lambda}$. The action of $\mathfrak{g}$ on each of them will be denoted $E_{+}$and $E_{-}$respectively. On their boundary $\partial \boldsymbol{S}_{ \pm}^{1 \oplus \lambda}=\boldsymbol{S}^{\lambda}$, the clutching $L$ is a $\mathbb{Z}_{m}$-isomorphism. We have the following precise definition.

Definition 3.1. 1. Clutching brackets: A clutching bracket over $S^{1 \oplus \lambda}$ (denoted by $\left.\left(n|L| E_{+} \mid E_{-}\right)\right)$consists of an integer $n$ called the dimension, a clutching function $L$, and actions $E_{ \pm}$of $\mathfrak{g}$ on $S_{ \pm}^{1 \oplus \lambda} \times \mathbb{C}^{n}$. Here $E_{ \pm}: D^{2} \rightarrow U(n)$ and $L: \partial D^{2} \rightarrow \boldsymbol{U}(n)$ are continuous functions such that

$$
\begin{aligned}
E_{-}(z) L(z) & =L(\bar{z}) E_{+}(z) & & \text { on } \partial D^{2}, \\
\left(E_{+}(\bar{a}) E_{+}(a)\right)^{q} & =\left(E_{-}(\bar{a}) E_{-}(a)\right)^{q}=I & & \text { for } a \in D^{2} .
\end{aligned}
$$

2. $D_{ \pm}(a)$ functions: Two auxiliary functions are defined for any clutching bracket $\left(n|L| E_{+} \mid E_{-}\right)$.

$$
D_{ \pm}(a)=E_{ \pm}(\bar{a}) E_{ \pm}(a) .
$$

They represent the $\mathbb{Z}_{q}$-action on the bundle.

Notice that: (a) $E_{ \pm}$are defined on $S_{ \pm}^{1 \oplus \lambda}$ if we identify $S_{ \pm}^{1 \oplus \lambda}$ with $D^{2}$. (b) On $\partial D^{2}$ we have $D_{-}(z) L(z)=L(z) D_{+}(z)$.

3. The associated bundle $\Theta\left(n|L| E_{+} \mid E_{-}\right)$: For a given clutching bracket $\left(n|L| E_{+} \mid E_{-}\right)$, we associate a $\mathbb{Z}_{m}$-bundle over $S^{1 \oplus \lambda}$ to it by using $L$ as a clutching function. Explicitly:

$$
\Theta\left(n|L| E_{+} \mid E_{-}\right)=S_{+}^{1 \oplus \lambda} \times \mathbb{C}^{n} \cup_{L} S_{-}^{1 \oplus \lambda} \times \mathbb{C}^{n} .
$$

The $\mathbb{Z}_{m}$-action is defined by:

$$
\begin{aligned}
& \boldsymbol{S}_{ \pm}^{1 \oplus \lambda} \times \mathbb{C}^{n} \stackrel{\mathfrak{g}}{\rightarrow} \boldsymbol{S}_{ \pm}^{1 \oplus \lambda} \times \mathbb{C}^{n}, \\
& (a, u) \rightarrow\left(\tau(a), E_{ \pm}(a) u\right) .
\end{aligned}
$$

4. Normal clutching brackets: $\left(n|L| E_{+} \mid E_{-}\right)$is normal if $L$ is constantly equal to the identity. For any normal clutching bracket we have

$$
E_{-}(z)=E_{-}(z) L(z)=L(\bar{z}) E_{+}(z)=E_{+}(z)
$$

on $\partial D^{2}$. Thus $E_{ \pm}$defines a continuous function $E$ over $S^{1 \oplus \lambda}$. Accordingly, $D_{ \pm}$defines a continuous function $D$ over $S^{1 \oplus \lambda}$ and $D(a)=E(\tau(a)) E(a)$ for $a \in S^{1 \oplus \lambda}$. We shall write a normal clutching bracket by $(n|I| E \mid E)$, where $E=E_{+}=E_{-}$.

5. Special clutching brackets: $\left(n|L| E_{+} \mid E_{-}\right)$is special if it is normal and the functions $D_{ \pm}$are constant on $D^{2}$.

6. Constant clutching bracket: $\left(n|L| E_{+} \mid E_{-}\right)$is a constant clutching bracket if it is special and the functions $E_{ \pm}$are constant.

7. Direct sum of clutching brackets: Just as in the case of bracket, we define the direct sum for any two clutching brackets.

$$
\left(n|L| E_{+} \mid E_{-}\right) \oplus\left(r|J| K_{+} \mid K_{-}\right)=\left(n+r|L \oplus J|(E \oplus K)_{+} \mid(E \oplus K)_{-}\right),
$$


where, by our convention,

$$
(E \oplus K)_{ \pm}=E_{ \pm} \oplus K_{ \pm}=\left(\begin{array}{cc}
E_{ \pm} & 0 \\
0 & K_{ \pm}
\end{array}\right) .
$$

8. Isomorphism of clutching brackets: $\Phi=\left\{\Phi_{ \pm}\right\}$is said to be an isomorphism between $\left(n|L| E_{+} \mid E_{-}\right)$and $\left(n|J| K_{+} \mid K_{-}\right)$if $\Phi_{ \pm}: D^{2} \rightarrow U(n)$ is continuous and

$$
\begin{aligned}
K_{ \pm}(a) \Phi_{ \pm}(a) & =\Phi_{ \pm}(\bar{a}) E_{ \pm}(a), \quad \text { on } D^{2}, \\
\Phi_{-}(z) L(z) & =J(z) \Phi_{+}(z), \quad \text { on } \partial D^{2} .
\end{aligned}
$$

We shall write $\left(n|L| E_{+} \mid E_{-}\right) \cong\left(n|J| K_{+} \mid K_{-}\right)$for isomorphic clutching brackets.

If both $(n|I| E \mid E)$ and $(n|I| K \mid K)$ are normal clutching brackets, then the function

$$
\Phi(a)= \begin{cases}\Phi_{+}(a), & a \in \boldsymbol{S}_{+}^{1 \oplus \lambda}, \\ \Phi_{-}(a), & a \in \boldsymbol{S}_{-}^{1 \oplus \lambda},\end{cases}
$$

is well defined and the equality

$$
K(a) \Phi(a)=\Phi(\bar{a}) E(a)
$$

holds.

9. Homotopy of clutching brackets: $\boldsymbol{H}=\left\{H_{ \pm}\right\}$is a homotopy between $\left(n|L| E_{+} \mid E_{-}\right)$and $\left(n|J| K_{+} \mid K_{-}\right)$if $\left(n|\Gamma| H_{+} \mid H_{-}\right)$is a clutching bracket over $[0,1] \times \boldsymbol{S}^{1 \oplus \lambda}$ such that

$$
\left.\left(n|\Gamma| H_{+} \mid H_{-}\right)\right|_{(0) \times S^{1 \oplus \lambda}}=\left(n|L| E_{+} \mid E_{-}\right),
$$

while

$$
\left.\left(n|\Gamma| H_{+} \mid H_{-}\right)\right|_{(\mathrm{i}) \times S^{1 \oplus \lambda}}=\left(n|J| K_{+} \mid K_{-}\right) .
$$

In particular, we have

$$
H_{ \pm}(0, a)=E_{ \pm}(a), \quad H_{ \pm}(1, a)=K_{ \pm}(a)
$$

for all $a \in D^{2}$, and

$$
\Gamma(0, z)=L(z), \quad \Gamma(1, z)=J(z)
$$

for all $z \in \partial D^{2}$.

We shall write $\left(n|L| E_{+} \mid E_{-}\right) \simeq\left(r|J| K_{+} \mid K_{-}\right)$is homotopic clutching brackets.

10. Stable homotopy (or stable isomorphism): Two clutching brackets are stably homotopic (or stably isomorphic) if they become homotopic (or isomorphic) after adding constant clutching brackets.

Proposition 3.2. 1. If ( $\left.n|L| E_{+} \mid E_{-}\right)$is a clutching bracket over $\boldsymbol{S}^{1 \oplus \lambda}$, then $L(z)$ is homotopic to a constant map. In particular, $\Theta\left(n|L| E_{+} \mid E_{-}\right)$is nonequivariantly trivial. Thus the restriction map $\widetilde{\boldsymbol{K}}_{\mathbb{Z}_{m}}\left(\boldsymbol{S}^{1 \oplus \lambda}\right) \rightarrow \widetilde{\boldsymbol{K}}\left(\boldsymbol{S}^{1 \oplus \lambda}\right)=\widetilde{\boldsymbol{K}}\left(\boldsymbol{S}^{2}\right)$ is zero.

2. If $\left(n|L| E_{+} \mid E_{-}\right)$is a constant clutching bracket over $\boldsymbol{S}^{1 \oplus \lambda}$, then $\boldsymbol{\Theta}\left(n|L| E_{+} \mid E_{-}\right)$is a trivial $\mathbb{Z}_{m}$-bundle over $\boldsymbol{S}^{1 \oplus \lambda}$.

3 .

$$
\begin{aligned}
& \boldsymbol{\Theta}\left(\left(n|L| E_{+} \mid E_{-}\right) \oplus\left(r|J| K_{+} \mid K_{-}\right)\right) \\
& \quad \cong \boldsymbol{\Theta}\left(n|L| E_{+} \mid E_{-}\right) \oplus \boldsymbol{\Theta}\left(r|J| K_{+} \mid K_{-}\right) .
\end{aligned}
$$

4. If $\Phi=\left\{\Phi_{ \pm}\right\}:\left(n|L| E_{+} \mid E_{-}\right) \cong\left(r|J| K_{+} \mid K_{-}\right)$is an isomorphism, then

$$
\Theta\left(n|L| E_{+} \mid E_{-}\right) \cong \Theta\left(r|J| K_{+} \mid K_{-}\right)
$$

as $\mathbb{Z}_{m}$-bundles over $\boldsymbol{S}^{1 \oplus \lambda}$. 
5. If $\left(n|L| E_{+} \mid E_{-}\right) \simeq\left(r|J| K_{+} \mid K_{-}\right)$by $H$, then

$$
\Theta\left(n|L| E_{+} \mid E_{-}\right) \cong \Theta\left(n|J| K_{+} \mid K_{-}\right)
$$

as $\mathbb{Z}_{m}$-bundles over $\boldsymbol{S}^{1 \oplus \lambda}$.

6. If we restrict the $\mathbb{Z}_{m}$-action on $\Theta\left(n|L| E_{+} \mid E_{-}\right)$to a $\mathbb{Z}_{q}$-action, we get a $\mathbb{Z}_{q}$-bundle $\boldsymbol{S}^{1 \oplus \lambda} \times \mathbb{C}^{n}$ over $\boldsymbol{S}^{1 \oplus \lambda}$. The $\mathbb{Z}_{q}$-action is then given by:

$$
\begin{gathered}
\boldsymbol{S}_{ \pm}^{1 \oplus \lambda} \times \mathbb{C}^{n} \stackrel{b=g^{2}}{\rightarrow} S_{ \pm}^{1 \oplus \lambda} \times \mathbb{C}^{n}, \\
(a, u) \rightarrow\left(a, D_{ \pm}(a) u\right) .
\end{gathered}
$$

7. Any $\mathbb{Z}_{m}$-bundle over $\boldsymbol{S}^{1 \oplus \lambda}$ is isomorphic to some $\Theta\left(n|L| E_{+} \mid E_{-}\right)$.

8. Any $\mathbb{Z}_{m}$-bundle over $S^{1 \oplus \lambda}$ is nonequivariantly trivial.

Proof. 1. By the definition of clutching bracket we have

$$
E_{-}(z) L(z)=L(\bar{z}) E_{+}(z)
$$

on $\partial D^{2}=S^{1}$. Since $E_{ \pm}$is defined on $D^{2},\left.E_{ \pm}\right|_{\partial D^{2}}$ is homotopic to $I$. Thus $L(z)$ is homotopic to $L(\bar{z})$. This implies that, in $\pi_{1}(U(n)) \cong \mathbb{Z}$, we have $L(z)=L(\bar{z})=-L(z)$, so that $L(z)=0$ in $\pi_{1}(U(n))$.

Since the clutching function $L$ is nullhomotopic, $\Theta\left(n|L| E_{+} \mid E_{-}\right)$is nonequivariantly trivial.

4. The isomorphism between $\Theta\left(n|L| E_{+} \mid E_{-}\right)$and $\Theta\left(r|J| K_{+} \mid K_{-}\right)$is given by

$$
\begin{gathered}
S_{ \pm}^{1 \oplus \lambda} \times \mathbb{C}^{n} \rightarrow S_{ \pm}^{1 \oplus \lambda} \times \mathbb{C}^{r}, \\
(a, u) \rightarrow\left(a, \Phi_{ \pm}(a) u\right) .
\end{gathered}
$$

5. As an immediate consequence of the definition, we have

$$
\left.\Theta\left(n|\Gamma| H_{+} \mid H_{-}\right)\right|_{(0) \times \boldsymbol{S}^{1 \oplus \lambda}}=\Theta\left(n|L| E_{+} \mid E_{-}\right)
$$

and

$$
\left.\Theta\left(n|\Gamma| H_{+} \mid H_{-}\right)\right|_{(1) \times S^{1 \oplus \lambda}}=\Theta\left(n|J| K_{+} \mid K_{-}\right)
$$

as $\mathbb{Z}_{m}$-bundles. Here $\Theta\left(n|\Gamma| H_{+} \mid H_{-}\right)$is the $\mathbb{Z}_{m}$-bundle over $[0,1] \times \boldsymbol{S}^{1 \oplus \lambda}$ defined by $\left(n|\Gamma| H_{+} \mid H_{-}\right)$. Thus

$$
\Theta\left(n|L| E_{+} \mid E_{-}\right) \cong \Theta\left(n|J| K_{+} \mid K_{-}\right)
$$

as $\mathbb{Z}_{m}$-bundles.

7. Since $S_{ \pm}^{1 \oplus \lambda}$ is $\mathbb{Z}_{m}$ contractible, each $\mathbb{Z}_{m}$-bundle $\eta$ over $S^{1 \oplus \lambda}$ will be trivial if restricted to $S_{ \pm}^{1 \oplus \lambda}$. Letting $L$ be the clutching function of $\eta$ and taking as $E_{ \pm}$the maps coming from the $\mathbb{Z}_{m}$-action on $S_{ \pm}^{1 \oplus \lambda}$ we get a clutching bracket. It is then easy to see that the bundle associated to this clutching bracket is isomorphic to $\eta$. Q.E.D.

Proposition 3.3. 1. Any clutching bracket $\left(n|L| E_{+} \mid E_{-}\right)$is homotopic to a normal clutching bracket $(n|I| K \mid K)$.

2. Let $(n|I| K \mid K)$ and $(n|I| E \mid E)$ be normal clutching brackets. If

$$
K(\tau(a)) K(a)=E(\tau(a)) E(a)
$$

for all $a \in S^{1 \oplus \lambda}$, and

then

$$
\left.E\right|_{\Delta_{+} \cap \Delta_{-}}=\left.K\right|_{\Delta_{+} \cap \Delta_{-}},
$$

$$
(n|I| E \mid E) \cong(n|I| K \mid K) .
$$


Proof. 1. By Proposition 2.2, we know that $L(z)$ is homotopic to $I$. Take an extension $F$ of $L$ over $D^{2}$, that is, $F: D^{2} \rightarrow U(n)$ with $\left.F\right|_{\partial D^{2}}=L, F(0)=I$. Now define

$$
\begin{aligned}
H_{+}(t, a) & =E_{+}(a), \\
H_{-}(t, a) & =F(\bar{a})^{-1} F(t \bar{a}) E_{-}(a) F(t a)^{-1} F(a), \\
\Gamma(t, z) & =L(z)^{-1} F(t z) L(z),
\end{aligned}
$$

for $a \in D^{2}, t \in[0,1]$, and $z \in \partial D^{2}$.

Then $\left(n|\Gamma| H_{+} \mid H_{-}\right)$gives a homotopy between

$$
\left(n|\Gamma(0, z)| H_{0+} \mid H_{0_{-}}\right) \quad \text { and }\left(n|\Gamma(1, z)| H_{1_{+}} \mid H_{1_{-}}\right)=\left(n|L| E_{+} \mid E_{-}\right) .
$$

Since $\Gamma(0, z)=F(0)=I,\left(n|\Gamma(0, z)| H_{0+} \mid H_{0_{-}}\right)$is normal. In particular, $\Theta\left(n|L| E_{+} \mid E_{-}\right) \cong \Theta\left(n|\Gamma(0, z)| H_{0+} \mid H_{0-}\right)$.

2. Define

$$
\Phi(a)= \begin{cases}I, & a \in \Delta_{+}, \\ K(\tau(a)) E(\tau(a))^{-1}, & a \in \Delta_{-} .\end{cases}
$$

It is easy to check that $\Phi$ is well defined and

$$
K(a) \Phi(a)=\Phi(\tau(a)) E(a)
$$

for $a \in S^{1 \oplus \lambda}$. $\Phi$ gives the desired isomorphism.

Lemma 3.4. Suppose $(n|I| K \mid K)$ is a special clutching bracket; i.e., $K(\tau(a)) K(a)$ is constant.

1. If $(n|I| E \mid E)$ is another special clutching bracket with

$$
\left.E\right|_{\Delta_{+} \cap \Delta_{-}}=\left.K\right|_{\Delta_{+} \cap \Delta_{-}} .
$$

Then

$$
(n|I| E \mid E) \cong(n|I| K \mid K) .
$$

2. $(n|I| K \mid K)$ is stably homotopic to a special clutching bracket $(n|I| F \mid F)$ with $\left.F\right|_{\Delta_{+} \cap \Delta_{-}}$constant.

3. $(n|I| K \mid K)$ is stably homotopic to a constant clutching bracket.

4. $\Theta(n|I| K \mid K)$ is stably trivial.

Proof. 1. Follows from Proposition 3.3.

2. First of all, $\boldsymbol{S}^{1}=\Delta_{+} \cap \Delta_{-} \hookrightarrow S^{1 \oplus \lambda}$ has trivial $\mathbb{Z}_{m}$-action; i.e., $\tau(z)=z$ for $z \in S^{1}$. Since $(n|I| K \mid K)$ is special, we have that

$$
K(z) K(\tau(z))=K(z)^{2}=D_{0}
$$

is constant and $D_{0}$ is of the form $T^{-1} \Lambda_{0} T$ for some $T \in U(n)$, with $\Lambda_{0}$ diagonal and $\Lambda_{0}^{q}=D_{0}^{q}=I$.

Let $\eta=S^{1} \times \mathbb{C}^{n}$ be the bundle over $S^{1}$ with $\mathbb{Z}_{m}$-action defined by

$$
\mathfrak{g}(z, u)=(z, K(z) u) .
$$

Since $\widetilde{\boldsymbol{K}}\left(\boldsymbol{S}^{1}\right)=0$, there are representations $\boldsymbol{V}, \boldsymbol{W}$ of $\mathbb{Z}_{m}$ such that $\eta \oplus \boldsymbol{V} \cong \boldsymbol{W}$ as $\mathbb{Z}_{m}$-bundles over $S^{1}$, say via $\Phi$. Suppose that the action of $\mathfrak{g}$ on $V$ and $W$ is given respectively by diagonal matrices $\Lambda_{1}$ and $\Lambda_{2}$. Since $\Phi$ is $\mathbb{Z}_{m^{-}}$ equivariant we have

$$
\Phi(z)\left(\begin{array}{cc}
K(z) & 0 \\
0 & \Lambda_{1}
\end{array}\right)=\Lambda_{2} \Phi(z)
$$


Hence,

(1) $\quad\left(\begin{array}{cc}K(z) & 0 \\ 0 & \Lambda_{1}\end{array}\right)=\Phi^{-1}(z) \Lambda_{2} \Phi(z)$,

(2) $\left(\begin{array}{cc}K^{2}(z) & 0 \\ 0 & \Lambda_{1}^{2}\end{array}\right)=\Phi^{-1}(z) \Lambda_{2}^{2} \Phi(z)=\left(\begin{array}{cc}D_{0} & 0 \\ 0 & \Lambda_{1}^{2}\end{array}\right)=S^{-1}\left(\begin{array}{cc}\Lambda_{0} & 0 \\ 0 & \Lambda_{1}^{2}\end{array}\right) S$,

where

$$
S=\left(\begin{array}{ll}
T & 0 \\
0 & I
\end{array}\right)
$$

In particular, $\Lambda_{2}^{2}$ is similar to

$$
\left(\begin{array}{cc}
\Lambda_{0} & 0 \\
0 & \Lambda_{1}^{2}
\end{array}\right)
$$

Since both matrices are diagonal, we may assume

$$
\Lambda_{2}^{2}=\left(\begin{array}{cc}
\Lambda_{0} & 0 \\
0 & \Lambda_{1}^{2}
\end{array}\right)=\Lambda
$$

(after some permutations of rows and columns). Then (2) becomes

$$
\Phi^{-1}(z) \Lambda \Phi(z)=S^{-1} \Lambda S .
$$

If we put $\Phi_{1}(z)=\Phi(z) S^{-1}$, then $\Phi_{1}(z) \Lambda=\Lambda \Phi_{1}(z)$. Suppose that

$$
\Lambda=\left(\begin{array}{ccc}
\lambda_{1} I_{1} & \cdots & 0 \\
\vdots & \ddots & \vdots \\
0 & \cdots & \lambda_{k} I_{k}
\end{array}\right),
$$

where $I_{i}$ are identity matrices, and $\lambda_{i} \neq \lambda_{j}$ if $i \neq j$. Since $\Phi_{1}(z) \Lambda=\Lambda \Phi_{1}(z)$, $\Phi_{1}(z)$ must be of the form

$$
\Phi_{1}(z)=\left(\begin{array}{ccc}
\mu_{1}(z) & \cdots & 0 \\
\vdots & \cdots & \vdots \\
0 & \cdots & \mu_{k}(z)
\end{array}\right),
$$

where $\mu_{i}: S^{1} \rightarrow \boldsymbol{U}\left(n_{i}\right)$. By Lemma 2.5 of $\S 2$ we may assume that

$$
\mu_{i} \simeq\left(\begin{array}{cc}
z^{t_{i}} & 0 \\
0 & I
\end{array}\right), \quad t_{i} \in \mathbb{Z}
$$

Then

$$
\left(\begin{array}{cc}
z^{-t_{i}} & 0 \\
0 & I
\end{array}\right) \cdot \mu_{i} \simeq I
$$

Let $r_{i}: D^{2} \rightarrow \boldsymbol{U}\left(n_{i}\right)$ be an extension of

$$
\left(\begin{array}{cc}
z^{-t_{i}} & 0 \\
0 & I
\end{array}\right) \cdot \mu_{i} \simeq I
$$

to all of $D^{2}$ with $r_{i}(0)=I_{i}$. Define, for all $a \in D^{2}$,

$$
\Psi(a)=\left(\begin{array}{ccc}
r_{1}(a) & \cdots & 0 \\
\vdots & \ddots & \vdots \\
0 & \cdots & r_{k}(a)
\end{array}\right) .
$$


Let

Then

$$
D_{i}(z)=\left(\begin{array}{cc}
z^{-t_{i}} & 0 \\
0 & I
\end{array}\right)
$$

$$
\begin{aligned}
\Psi(z) & =\left(\begin{array}{ccc}
D_{1}(z) & \cdots & 0 \\
\vdots & \ddots & \vdots \\
0 & \cdots & D_{k}(z)
\end{array}\right) \Phi_{1}(z) \\
& =\left(\begin{array}{ccc}
D_{1}(z) & \cdots & 0 \\
\vdots & \ddots & \vdots \\
0 & \cdots & D_{k}(z)
\end{array}\right) \Phi(z) S^{-1} \\
& =\boldsymbol{D} \Phi(z) S^{-1}
\end{aligned}
$$

where

$$
\boldsymbol{D}=\left(\begin{array}{ccc}
D_{1}(z) & \cdots & 0 \\
\vdots & \ddots & \vdots \\
0 & \cdots & D_{k}(z)
\end{array}\right)
$$

We have $\Psi(a) \Lambda=\Lambda \Psi(a)$, as can be easily verified. Define, for all $a \in S^{1 \oplus \lambda}$,

$$
\widetilde{\Psi}(a)= \begin{cases}\Psi(a), & a \in \Delta_{+}, \\ \Psi(a), & a \in \Delta_{-},\end{cases}
$$

and

$$
F(a) S^{-1} \widetilde{\Psi}(a) S\left(\begin{array}{cc}
K(a) & 0 \\
0 & \Lambda_{1}
\end{array}\right) S^{-1} \widetilde{\Psi}^{-1}(\tau(a)) S .
$$

Then $\tilde{\Psi} \Lambda=\Lambda \tilde{\Psi}$. Hence,

$$
\begin{aligned}
F(\tau(a)) F(a) & =S^{-1} \widetilde{\Psi}(\tau(a)) S\left(\begin{array}{cc}
K(\tau(a)) K(a) & 0 \\
0 & \Lambda_{1}^{2}
\end{array}\right) S^{-1} \tilde{\Psi}^{-1}(\tau(a)) S \\
& =S^{-1} \tilde{\Psi}(\tau(a)) \Lambda \tilde{\Psi}^{-1}(\tau(a)) S=S^{-1} \Lambda S=\text { constant. }
\end{aligned}
$$

This proves that $F$ is special. Using (1) and (3) we find

$$
\begin{aligned}
\left.F\right|_{\Delta_{+} \cap \Delta_{-}} & =S^{-1} \tilde{\Psi}(z) S\left(\begin{array}{cc}
K(z) & 0 \\
0 & \Lambda_{1}
\end{array}\right) S^{-1} \tilde{\Psi}^{-1}(z) S \\
& =S^{-1} \Psi(z) S\left(\begin{array}{cc}
K(z) & 0 \\
0 & \Lambda_{1}
\end{array}\right) S^{-1} \Psi^{-1}(z) S \\
& =S^{-1} D \Phi(z)\left(\begin{array}{cc}
K(z) & 0 \\
0 & \Lambda_{1}
\end{array}\right) \Phi^{-1}(z) D^{-1} S \\
& =S^{-1} D \Lambda_{2} D^{-1} S \\
& =S^{-1} \Lambda_{2} S=\text { constant, with } \Lambda_{2} \text { diagonal. }
\end{aligned}
$$

Now define

$$
E(t, a)=S^{-1} \widetilde{\Psi}_{t}(a) S\left(\begin{array}{cc}
K(a) & 0 \\
0 & \Lambda_{1}
\end{array}\right) S^{-1} \tilde{\Psi}_{t}^{-1}(\tau(a)) S
$$

with

$$
\widetilde{\Psi}_{t}(a)= \begin{cases}\Psi(t a), & a \in \Delta_{+} \\ \Psi(t a), & a \in \Delta_{-}\end{cases}
$$


Then

$$
E(0, a)=\left(\begin{array}{cc}
K(a) & 0 \\
0 & \Lambda_{1}
\end{array}\right)
$$

and $E(1, a)=F(a)$.

Thus $E(t, a)$ is a homotopy between

$$
(r|I| E(0, a) \mid E(0, a))=(n|I| K \mid K) \oplus\left(l|I| \Lambda_{1} \mid \Lambda_{1}\right)
$$

and

$$
(r|I| E(1, a) \mid E(1, a))=(r|I| F \mid F)
$$

with $\left(l|I| \Lambda_{1} \mid \Lambda_{1}\right)$ a constant clutching bracket, $(r|I| F \mid F)$ special, and $\left.E\right|_{\Delta_{+} \cap \Delta_{-}}$ constant. This proves 2 .

3. If $(n|I| F \mid F)$ is a constant clutching bracket with $\left.F\right|_{\Delta_{+} \cap \Delta_{-}}$a constant $F_{0}$, then $\left(n|I| F_{0} \mid F_{0}\right)$ is a constant clutching bracket with

$$
\left.F_{0}\right|_{\Delta_{+} \cap \Delta_{-}}=\left.F\right|_{\Delta_{+} \cap \Delta_{-}} \text {. }
$$

By 1 ,

$$
(n|I| F \mid F) \cong\left(n|I| F_{0} \mid F_{0}\right) \text {. Q.E.D. }
$$

Lemma 3.5. Suppose that $\eta$ is a $\mathbb{Z}_{m}$-bundle over $\boldsymbol{S}^{1 \oplus \lambda}$ such that $\eta$ restricts to 0 in $\widetilde{\boldsymbol{K}}_{\mathbb{Z}_{q}}\left(\boldsymbol{S}^{1 \oplus \lambda}\right)$. Then $\eta=0$ in $\widetilde{\boldsymbol{K}}_{\mathbb{Z}_{m}}\left(\boldsymbol{S}^{1 \oplus \lambda}\right)$. In other words res : $\widetilde{\boldsymbol{K}}_{\mathbb{Z}_{m}}\left(\boldsymbol{S}^{1 \oplus \lambda}\right) \hookrightarrow$ $\widetilde{\boldsymbol{K}}_{\mathbb{Z}_{q}}\left(\boldsymbol{S}^{1 \oplus \lambda}\right)=\widetilde{\boldsymbol{K}}_{\mathbb{Z}_{q}}\left(\boldsymbol{S}^{2}\right)$ is an injection.

Proof. res $\eta=0$ means that there are representations $\boldsymbol{V}$ and $\boldsymbol{W}$ of $\mathbb{Z}_{q}$ such that

$$
\text { res } \eta \oplus \boldsymbol{W} \equiv \boldsymbol{V}
$$

as $\mathbb{Z}_{q}$-bundles, say via $\Phi$. According to Proposition 3.3, we may pick a normal clutching bracket $(n|I| K \mid K)$ such that

$$
\eta=\Theta(n|I| K \mid K) \text {. }
$$

We know by Proposition 3.2 that the action of $\mathfrak{b} \in \mathbb{Z}_{q}$ on res $\eta$ is defined by $D(a)=K(\tau(a)) K(a)$. Since $\Phi$ is $\mathbb{Z}_{q}$-equivariant,

$$
\Phi(a)\left(\begin{array}{cc}
D(a) & 0 \\
0 & \Lambda_{0}
\end{array}\right)=\Lambda \Phi(a),
$$

where $\Lambda$ and $\Lambda_{0}$ are diagonal matrices determining the actions of $\mathfrak{b}=\mathfrak{g}^{2} \in \mathbb{Z}_{q}$ on $\boldsymbol{W}$ and $\boldsymbol{V}$, respectively. Now define

$$
E(a)=\Phi(a)\left(\begin{array}{cc}
K(a) & 0 \\
0 & \Lambda_{0}^{1 / 2}
\end{array}\right) \Phi^{-1}(\tau(a)) .
$$

Then

$$
E(\tau(a)) E(a)=\Lambda=\text { constant. }
$$

Thus the bracket $(r|I| E \mid E)$ is special. In particular,

$$
\Theta(r|I| E \mid E)=0 \in \widetilde{\boldsymbol{K}}_{\mathbb{Z}_{m}}\left(\boldsymbol{S}^{1 \oplus \lambda}\right),
$$

by Corollary 3.4. Let $\pi(a)=\Phi(\tau(a))$ for $a \in S^{1 \oplus \lambda}$. Then

$$
E(a) \pi(a)=\pi(\tau(a))\left(\begin{array}{cc}
K(a) & 0 \\
0 & \Lambda_{0}
\end{array}\right) .
$$


This implies that $\pi$ is an isomorphism between

$$
\left(r|I|\left(\begin{array}{cc}
K & 0 \\
0 & \Lambda_{0}
\end{array}\right) !\left(\begin{array}{cc}
K & 0 \\
0 & \Lambda_{0}
\end{array}\right)\right)
$$

and

$$
(r|I| E \mid E) .
$$

Hence

$$
\begin{aligned}
\Theta\left(r|I|\left(\begin{array}{cc}
K & 0 \\
0 & \Lambda_{0}
\end{array}\right) \mid\left(\begin{array}{cc}
K & 0 \\
0 & \Lambda_{0}
\end{array}\right)\right) & =\Theta(n|I| K \mid K) \oplus \Theta\left(l|I| \Lambda_{0} \mid \Lambda_{0}\right) \\
& \cong \Theta(r|I| E \mid E) .
\end{aligned}
$$

Since $\left(l|I| \Lambda_{0} \mid \Lambda_{0}\right)$ is a constant clutching bracket, $\Theta\left(l|I| \Lambda_{0} \mid \Lambda_{0}\right)$ is a trivial $\mathbb{Z}_{m^{-}}$ bundle. This proves that

$$
\eta=\Theta(n|I| K \mid K)=0 \in \widetilde{K}_{\mathbb{Z}_{m}}\left(\boldsymbol{S}^{1 \oplus \lambda}\right) \text {. Q.E.D. }
$$

Definition 3.6. Elementary clutching brackets and elementary bundles:

1. Set $L(z)=\left(\begin{array}{l}z \\ 0\end{array} \frac{0}{z}\right)$ on $\partial D^{2} . L(z)$ is nullhomotopic. Hence it extends to all of $D^{2}$ and we may assume $L(0)=I$.

2. Let $\xi=e^{2 \pi i / m}$. For each $1 \leq k \leq m$ define

$$
E_{k+}(a)=\left(\begin{array}{cc}
1 & 0 \\
0 & \xi^{k}
\end{array}\right) \quad \text { for } a \in S_{+}^{1 \oplus \lambda}
$$

and

$$
E_{k-}(a)=L(\bar{a})\left(\begin{array}{cc}
1 & 0 \\
0 & \xi^{k}
\end{array}\right) L^{-1}(a) \quad \text { for } a \in S_{-}^{1 \oplus \lambda}
$$

Then

$$
D_{k+}(a)=E_{k+}(\tau(a)) E_{k+}(a)=\left(\begin{array}{cc}
1 & 0 \\
0 & \xi^{2 k}
\end{array}\right) \quad \text { for } a \in S_{+}^{1 \oplus \lambda},
$$

and

$$
D_{k-}(a)=E_{k-}(\tau(a)) E_{k-}(a)=L(a)\left(\begin{array}{cc}
1 & 0 \\
0 & \xi^{2 k}
\end{array}\right) L^{-1}(a) \quad \text { for } a \in S_{-}^{1 \oplus \lambda} .
$$

Each $\Theta\left(2|L| E_{k_{+}} \mid E_{k-}\right)$ is called an elementary clutching bracket.

3. The associated bundle $\delta_{k}=\Theta\left(2|I| E_{k+} \mid E_{k_{-}}\right)$is called an elementary bundle. We shall write $\delta$ for $\delta_{1}$. Q.E.D.

Proposition 3.7. 1. res: $\widetilde{\boldsymbol{K}}_{\mathbf{Z}_{m}}\left(\boldsymbol{S}^{1 \oplus \lambda}\right) \rightarrow \widetilde{\boldsymbol{K}}_{\mathbb{Z}_{q}}\left(\boldsymbol{S}^{1 \oplus \lambda}\right)$ maps $\delta_{k}$ to $\gamma \oplus \bar{\gamma} \otimes \chi_{2 k}$, where $\gamma$ is the Hopf bundle over $\boldsymbol{S}^{2}$ and $\bar{\gamma}$ its complex dual $(=-\gamma)$.

2. Let

$$
\begin{aligned}
\eta= & \left(n_{1} \gamma \otimes \chi_{2}\right) \oplus\left(n_{2} \gamma \otimes \chi_{4}\right) \oplus \cdots \oplus\left(n_{q} \gamma \otimes \chi_{2 q}\right) \\
& \oplus\left(p_{1} \bar{\gamma} \otimes \chi_{2}\right) \oplus\left(p_{2} \bar{\gamma} \otimes \chi_{4}\right) \oplus \cdots \oplus\left(p_{q} \bar{\gamma} \otimes \chi_{2 q}\right)
\end{aligned}
$$

in $\widetilde{\boldsymbol{K}}_{\mathrm{Z}_{q}}\left(\boldsymbol{S}^{1 \oplus \lambda}\right)$ with $n_{1}+\cdots+n_{q}=p_{1}+\cdots+p_{q}$. Then $\eta$ is in the subgroup generated by $\left\{\operatorname{res} \delta_{k}\right\}_{k=1}^{q}$.

3. res $\delta_{1}, \ldots$, res $\delta_{q}$ generate the image of res in $\widetilde{\boldsymbol{K}}_{\mathbf{Z}_{q}}\left(\boldsymbol{S}^{1 \oplus \lambda}\right)=\widetilde{\boldsymbol{K}}_{\mathbf{Z}_{q}}\left(\boldsymbol{S}^{2}\right)$.

4. The image of res: $\widetilde{\boldsymbol{K}}_{\mathbb{Z}_{m}}\left(\boldsymbol{S}^{1 \oplus \lambda}\right) \hookrightarrow \widetilde{\boldsymbol{K}}_{\mathbb{Z}_{q}}\left(\boldsymbol{S}^{1 \oplus \lambda}\right)$ is

$$
\operatorname{res}\left(\boldsymbol{R}\left(\mathbb{Z}_{m}\right) \delta_{1}\right)=\boldsymbol{R}\left(\mathbb{Z}_{q}\right) \operatorname{res} \delta_{1}=\boldsymbol{R}\left(\mathbb{Z}_{q}\right) \operatorname{res} \delta .
$$


Proof. 1. The action of $\mathbb{Z}_{q}$ on res $\delta_{k}$ is given by $D_{k \pm}(a)$. Since $\mathbb{Z}_{q}$ acts trivially on $S^{1 \oplus \lambda}$, res $\delta_{k}=\sum \operatorname{Hom}_{\mathbb{Z}_{q}}\left(\chi_{2 i}, \delta_{k}\right) \otimes \chi_{2 i}$. Because the action of $\mathbb{Z}_{q}$ on each fiber of res $\delta_{k}$ is $1+\chi_{2 k}$, an easy argument gives res $\delta_{k}=\gamma \oplus \bar{\gamma} \otimes \chi_{2 k}$. In particular,

$$
\begin{aligned}
\operatorname{res}\left(\delta_{k}-\delta_{k+1}\right) & =\bar{\gamma} \otimes \chi_{2 k} \oplus \gamma \otimes \chi_{2 k+2} \\
& =\gamma \otimes \chi_{2 k+2}-\gamma \otimes \chi_{2 k} .
\end{aligned}
$$

2.

$$
\begin{aligned}
\eta= & \left(n_{1}-p_{1}\right) \gamma \otimes \chi_{2} \oplus\left(n_{2}-p_{2}\right) \gamma \otimes \chi_{4} \oplus \cdots \oplus\left(n_{q}-p_{q}\right) \gamma \otimes \chi_{2 q} \\
= & \left(n_{1}-p_{1}\right)\left(\gamma \otimes \chi_{2}-\gamma \otimes \chi_{4}\right) \\
& \oplus\left(n_{1}-p_{1}+n_{2}-p_{2}\right)\left(\gamma \otimes \chi_{4}-\gamma \otimes \chi_{6}\right) \oplus \cdots \\
& \oplus\left(n_{1}-p_{1}+n_{2}-p_{2}+\cdots+n_{q-1}-p_{q-1}\right)\left(\gamma \otimes \chi_{2 q-2}-\gamma \otimes \chi_{2 q}\right) \\
& \oplus\left(n_{1}-p_{1}+\cdots+n_{q}-p_{q}\right) \gamma \otimes \chi_{2 q} \\
= & -\left[\left(n_{1}-p_{1}\right) \operatorname{res}\left(\delta_{1}-\delta_{2}\right)\right. \\
& \oplus\left(n_{1}-p_{1}+n_{2}-p_{2}\right) \operatorname{res}\left(\delta_{2}-\delta_{3}\right) \oplus \cdots \\
& \left.\oplus\left(n_{1}-p_{1}+n_{2}-p_{2}+\cdots+n_{q-1}-p_{q-1}\right) \operatorname{res}\left(\delta_{q-1}-\delta_{q}\right)\right]
\end{aligned}
$$

(because $n_{1}-p_{1}+\cdots+n_{q}-p_{q}=0$ ).

3. For any $\mathbb{Z}_{m}$-bundle $\eta$ over $S^{1 \oplus \lambda}$,

$$
\begin{aligned}
\text { res } \eta \oplus \boldsymbol{W} \equiv & \boldsymbol{V} \oplus\left(n_{1} \gamma \otimes \chi_{2}\right) \oplus\left(n_{2} \gamma \otimes \chi_{4}\right) \oplus \cdots \oplus\left(n_{q} \gamma \otimes \chi_{2 q}\right) \\
& \oplus\left(p_{1} \bar{\gamma} \otimes \chi_{2}\right) \oplus\left(p_{2} \bar{\gamma} \otimes \chi_{4}\right) \oplus \cdots \oplus\left(p_{q} \bar{\gamma} \otimes \chi_{2 q}\right)
\end{aligned}
$$

as $\mathbb{Z}_{q}$-bundles for some representations $\boldsymbol{V}$ and $\boldsymbol{W}$ of $\mathbb{Z}_{q}$. By 1 of Proposition $3.2, \eta$ is nonequivariantly trivial. Thus, nonequivariantly,

$$
\begin{aligned}
& \left(n_{1} \gamma \otimes \chi_{2}\right) \oplus\left(n_{2} \gamma \otimes \chi_{4}\right) \oplus \cdots \oplus\left(n_{q} \gamma \otimes \chi_{2 q}\right) \\
& \quad \oplus\left(p_{1} \bar{\gamma} \otimes \chi_{2}\right) \oplus\left(p_{2} \bar{\gamma} \otimes \chi_{4}\right) \oplus \cdots \oplus\left(p_{q} \bar{\gamma} \otimes \chi_{2 q}\right) \\
& \quad \doteq\left(n_{1}-p_{1}+\cdots+n_{q}-p_{q}\right) \gamma
\end{aligned}
$$

is trivial. Therefore,

$$
n_{1}-p_{1}+\cdots+n_{q}-p_{q}=0
$$

i.e.,

4.

$$
n_{1}+\cdots+n_{q}=p_{1}+\cdots+p_{q}
$$

$$
\begin{aligned}
\operatorname{res} \delta_{k} & =\left(\operatorname{res} \delta_{1}\right) \otimes \chi_{2} \oplus\left(\operatorname{res} \delta_{1}\right) \otimes \chi_{4} \oplus \cdots \oplus\left(\operatorname{res} \delta_{1}\right) \otimes \chi_{2 k-2} \\
& =\left(\operatorname{res} \delta_{1}\right) \otimes\left(\chi_{2}+\chi_{4}+\cdots+\chi_{2 k-2}\right) \\
& =\operatorname{res}\left(\delta_{1} \otimes\left(\chi_{1}+\cdots+\chi_{k-1}\right)\right) .
\end{aligned}
$$

Theorem B.

$$
\widetilde{\boldsymbol{K}}_{\mathbb{Z}_{m}}\left(\boldsymbol{S}^{1 \oplus \lambda}\right) \cong \boldsymbol{R}\left(\mathbb{Z}_{q}\right)\left(\gamma \oplus \bar{\gamma} \otimes \chi_{2}\right) \cong \boldsymbol{R}\left(\mathbb{Z}_{q}\right)\left(\chi_{2}-\chi_{0}\right)
$$

as ideals, where $\chi_{2}$ is the one-dimensional complex representation of $\mathbb{Z}_{q}$ defined by $b z=e^{\pi i / q} z$, and $b \in \mathbb{Z}_{q}$ is the generator and $z \in \mathbb{C}$. In particular, $\widetilde{\boldsymbol{K}}_{\mathbb{Z}_{2}}\left(\boldsymbol{S}^{1 \oplus \lambda}\right)=0$.

Proof. Theorem B follows from, Lemma 3.5 and Proposition 3.7. 


\section{The proofs of Lemma D AND Theorem E}

We proved in $\S \S 2$ and 3 that the coefficient ring for any cyclic group is torsionfree. This determines the nontorsion part of the coefficient group for any group $G$, since the rationalization of $K_{G}$ is determined by the restriction to the cyclic subgroups of $G$.

The main tool we shall use is the fact that the torsion part is completely determined by its restriction to the elementary subgroups. The strategy, therefore, is to prove the corresponding results for the elementary groups.

Definition 4.1. Recall that a group $G=\mathbb{Z}_{m} \times P$ is an elementary group if $P$ is a $p$-group and the order of $P$ is prime to $m$.

Lemma 4.2. Suppose $G=\mathbb{Z}_{m} \times P$. Let $\mathrm{V}$ be an irreducible pure real representation of $G$. Then $\mathrm{V} \cong \mu \otimes \mathrm{U}$ for some one-dimensional pure real irreducible representation $\mu$ of $\mathbb{Z}_{m}$ and some pure real irreducible representation $\mathrm{U}$ of $P$.

Proof. Since V is pure real irreducible, by standard results in representation theory we know that $\mathrm{v} \otimes \mathbb{C}$ is an irreducible complex representation of $G$. Now suppose $\mathrm{V} \otimes \mathbb{C}=\beta \otimes W$, where $\beta$ is an irreducible complex representation of $P$. (We do not know yet if $\mathrm{v}$ can be written as a tensor product of two real representations.) Since $\mathrm{V} \otimes \mathbb{C}$ is irreducible, it has a nonsingular symmetric bilinear $\mathbb{Z}_{m} \times P$-invariant form $\langle$,$\rangle . Since \mathbb{Z}_{m}$ is cyclic, $\beta$ is one-dimensional. Thus, as a complex space, $\boldsymbol{W} \doteq \mathrm{v} \otimes \mathbb{C}$. Therefore, $\langle$,$\rangle is nonsingular on$ $\boldsymbol{W} \doteq \mathrm{V} \otimes \mathbb{C}$ and, when restricted to $P$, is a nonsingular symmetric bilinear $P$-invariant form. This implies that $W=\mathrm{U} \otimes \mathbb{C}$ for some irreducible real representation $U$ of $P$. In particular, $\bar{W}=W$. But then

$$
\mathrm{V} \otimes \mathbb{C}=\overline{\mathrm{V} \otimes \mathbb{C}}=\beta \otimes W=\overline{\beta \otimes W}=\bar{\beta} \otimes \bar{W}=\bar{\beta} \otimes W .
$$

It follows that $\beta \otimes W=\bar{\beta} \otimes W$. This implies $\beta=\bar{\beta}$. Thus we have

$$
\beta= \begin{cases}1 & \text { if } m=\text { odd }, \\ 1 \text { or possibly } \lambda \otimes \mathbb{C} & \text { if } m=\text { even. }\end{cases}
$$

This proves that $\beta=\mu \otimes \mathbb{C}$ for a one-dimensional real representation $\mu$ of $\mathbb{Z}_{m}$ defined by

$$
\mu= \begin{cases}1 & \text { if } m=\text { odd } \\ 1 \text { or possibly }-1 & \text { if } m=\text { even }\end{cases}
$$

Hence,

$$
\mathrm{V} \otimes \mathbb{C}=\boldsymbol{\beta} \otimes \boldsymbol{W}=(\mu \otimes \mathbb{C}) \otimes(\mathrm{U} \otimes \mathbb{C})=\mu \otimes \mathrm{U} \otimes \mathbb{C}
$$

and therefore, $\mathrm{V}=\mu \otimes \mathrm{U}$. Since $\mathrm{V}$ contains no complex representations of $G$, $U$ contains no complex representations of $P$ and $W=U \otimes \mathbb{C}$ is irreducible. Q.E.D.

Lemma D. Suppose $G=\mathbb{Z}_{m} \times P$, where $P$ is a p-group. Let $\mathrm{V}$ be a pure real representation of $G$.

1. If $p$ is odd, then $\mathrm{V}=\alpha \otimes 1$. Here $\alpha$ is a pure real representation of $\mathbb{Z}_{m}$.

2. If $m$ is odd, then $\mathrm{V}=1 \otimes \alpha$. Here $\alpha$ is a pure real representation of $P$. In particular, if $G$ is an elementary group and $P$ a 2-group, then every pure real representation is of the form $1 \otimes \alpha$. 
Proof. Suppose $\mathrm{V}=\mathrm{v}_{1} \oplus \mathrm{v}_{2} \oplus \cdots \oplus \mathrm{v}_{k}$, where each $\mathrm{V}_{i}$ is a real irreducible representation of $G$. Because $\mathrm{V}$ contains no complex representation, neither do any of the $\mathrm{V}_{i}$. By Lemma 4.2, $\mathrm{v}_{i}=\mu_{i} \otimes \mathrm{U}_{i}$.

1. This is because an odd order group has no nontrivial irreducible representations of real type.

2. Since $m$ is odd, all $\mu_{i}$ are trivial. $\mathrm{V}=1 \otimes\left(\sum_{i} \mathrm{U}_{i}\right)=1 \otimes \alpha$, where $\alpha=\sum_{i} \mathrm{U}_{i}$.

Theorem E. 1. If $P$ is a p-group with $p$ odd, then $\widetilde{\boldsymbol{K}}_{\mathbb{Z}_{m} \times P}\left(\boldsymbol{S}^{*}\right)$ is torsion-free.

2. If $Q$ is a 2-group, then $\widetilde{\boldsymbol{K}}_{\mathbb{Z}_{m} \times Q}\left(\boldsymbol{S}^{*}\right)$ can only have 2-torsion.

Proof. 1. By 1 of Lemma D, we have to prove that if $\alpha$ is either $\lambda$ or $1 \oplus \lambda$, where $\lambda$ is the nontrivial one-dimensional real representation of $\mathbb{Z}_{m}$, then $\widetilde{\boldsymbol{K}}_{\mathbb{Z}_{m} \times P}\left(\boldsymbol{S}^{\alpha \otimes 1}\right)$ is torsion-free.

But we can apply a generalization of a Graeme Segal proposition [7, Proposition 2.2]:

$$
\boldsymbol{K}_{G \times H}(X) \cong \boldsymbol{K}_{H}(X) \otimes \boldsymbol{R}(G)
$$

for any $G \times H$-space $X$ with $G$ acting trivially. We obtained:

$$
\boldsymbol{K}_{\mathbb{Z}_{m} \times P}\left(\boldsymbol{S}^{\alpha \otimes 1}\right) \cong \boldsymbol{K}_{\mathbb{Z}_{m}}\left(\boldsymbol{S}^{\alpha}\right) \otimes \boldsymbol{R}(P) .
$$

The latter is torsion-free by Theorems A and B.

2. The proof is identical. We have the following isomorphism:

$$
\boldsymbol{K}_{\mathbb{Z}_{m} \times Q}\left(\boldsymbol{S}^{1 \otimes \alpha}\right) \cong \boldsymbol{R}\left(\mathbb{Z}_{m}\right) \otimes \boldsymbol{K}_{\times Q}\left(\boldsymbol{S}^{\alpha}\right)
$$

for $\alpha$ any real representation of $Q$.

The group $\boldsymbol{K}_{Q}\left(\boldsymbol{S}^{\alpha}\right)$ can only have 2-torsions because of the Proposition 1 in the introduction.

\section{REFERENCES}

1. W. Burnside, Theory of groups of finite order, Cambridge Univ. Press, 1911.

2. Stefan Jackowski, Equivariant $K$-theory and cyclic subgroups, London Math. Soc. Lecture Notes Ser., vol. 26, Cambridge Univ. Press, 1977, pp. 76-91.

3. G. Lewis, J. P. May, and J. E. McClure, Ordinary $R O(G)$-graded cohomology, Bull. Amer. Math. Soc. (N.S.) 4 (1981), 208-212.

4. G. Lewis, Jr., J. P. May, and M. Steinberg (with contributions by J. E. McClure), Equivariant stable homotopy theory, Lecture Notes in Math., vol. 1213, Springer-Verlag, 1986.

5. James E. McClure, Restriction maps in equivariant $K$-theory, Topology 25 (1986), 399-409.

6. Ryszard L. Rubinsztein, Restriction of equivariant $K$-theory, Bull. Acad. Polon. Sci. Sér. Sci. Math. 29 (1981, no. 5-6).

7. G. B. Segal, Equivariant K-theory, Publ. Math. Inst. Hautes Étude Sci. 34 (1968), 129-151.

8. J.-P. Serre, Linear representations of finite groups, Springer-Verlag, 1977.

Division of Science and Mathematics, University of Minnesota, Morris, Minnesota 56267

E-mail address: yang@cda.mrs.umn.edu 